\title{
Van Hove Limit for Infinite Systems
}

\author{
David Taj
}

\begin{abstract}
We study the van Hove limit for master equations on a Banach space, and propose a contraction semigroup as limit dynamics. The generator has a Lindblad form if specialized to $C^{*}$-algebras, is always well defined irrespectively of the subsystem spectrum, includes first-order contributions, and returns Davies averaged generator, when the latter is defined. The theory is applied to the case of a free particle in contact with a heat bath.
\end{abstract}

\section{Introduction}

The Van Hove limit [1], also referred to as weak-coupling limit, amounts to study the time evolution of a perturbed hamiltonian system on the $\lambda^{2} t$ time scale, as the coupling constant $\lambda$ goes to zero. It has long been rigorously known $[2,3]$ that the time evolution of a finite (or discrete) quantum system, interacting with an external steady environment, becomes markovian in such circumstances, as described by the celebrated Fermi Golden Rule $[4,5]$.

However, nowadays technologies often require decay times and steadystate analysis [6] for infinitely extended open quantum systems [7], i.e. with continuous, and even mixed spectrum. Although phenomenological markovian laws for such systems have been studied (see e.g. [8-10]), a general recipe to construct a proper markovian generator in the van Hove limit, given the hamiltonian perturbation, is lacking.

This appears to be because the limit dynamics that has been found [3] fails to provide a positive [11] (or even contractive [12]) evolution in general; moreover, the time averaging procedure [2], successfully employed to remedy for this in the discrete case, is not well defined anymore in the continuum. As a consequence, the resulting markovian law cannot be adopted as a consistent physical model per se, and large time behavior and steadiness cannot be addressed.

Here, we propose a semigroup that (i) is contractive, (ii) is always well defined irrespectively of the subsystem spectrum, (iii) includes first-order 
dynamics, (iv) approaches the exact projected evolution in the van Hove limit, and upon specializing to operator algebras, (v) is (completely) positive. Also, (vi) Davies averaged generator is recovered (in a slightly generalized form including possible first-order dynamics) in case of discrete spectrum.

Formalizing some ideas of a previous work of ours [13], as well as introducing new ones, on rigorous grounds, the proposed Quantum Fokker-Planck Equation in (16) is constructed through a "dynamical time averaging" that a posteriori mimics the procedure employed in [2], but differs from the latter, in that it scales with the coupling constant.

Finally, we apply the theory to the case of free particle in contact with a heat bath through energy-energy couplings, and find sufficient conditions, different with respect to the discrete case, to prove that thermal distribution of diagonal observables (i.e. affiliated to the hamiltonian of the small system) is stationary. The analysis is far from being exhaustive and indicates that a plethora of new possibilities opens up.

\section{General Framework}

We briefly report from [3] the general framework we will be involved with. We suppose that $P_{0}$ is a linear projection on a Banach space $\mathcal{B}$ (that represents some global system), put $P_{1}=1-P_{0}$ and $\mathcal{B}_{i}=P_{i} \mathcal{B}$, so that $\mathcal{B}=\mathcal{B}_{0} \oplus \mathcal{B}_{1}$, and we take $\mathcal{B}_{0}$ to be the subsystem of interest. We suppose that $Z$ is the (densely defined) generator of a strongly continuous one-parameter group of isometries $U_{t}$ on $\mathcal{B}$ with $U_{t} P_{0}=P_{0} U_{t}$ for all $t \in \mathbb{R}$, or equivalently $\left[Z, P_{0}\right]=0$, and put $Z_{i}=P_{i} Z$. We suppose that $A$ is a bounded perturbation of $Z$ and put $A_{i j}=$ $P_{i} A P_{j}$. We let $U_{t}^{\lambda}$ be the one-parameter group generated by $\left(Z+\lambda A_{00}+\lambda A_{11}\right)$ so that $U_{t}^{\lambda} P_{0}=P_{0} U_{t}^{\lambda}$ for all $t \in \mathbb{R}$, and let $V_{t}^{\lambda}$ be the one-parameter group generated by $Z+\lambda A$. Then putting $X_{t}^{\lambda}=P_{0} U_{t}^{\lambda}$, and defining the projected evolution as $W_{t}^{\lambda}=P_{0} V_{t}^{\lambda} P_{0}$, one obtains the all important closed and exact integral master equation:

$$
W_{t}^{\lambda}=X_{t}^{\lambda}+\lambda^{2} \int_{0}^{t} \mathrm{~d} s \int_{0}^{s} \mathrm{~d} u X_{t-s}^{\lambda} A_{01} U_{s-u}^{\lambda} A_{10} W_{u}^{\lambda},
$$

named after Nakajima, Prigogine, Resibois, and Zwanzig [14,15].

Now assume that $X_{t}^{\lambda}$ is a one-parameter group of isometries (see Lemma 1.1 in [3]). Then, changing variables in the integral in (1) to $x=s-u, \sigma=\lambda^{2} u$ and introducing the time rescaled (and $A_{00}$-renormalized) interaction picture evolution $W_{\tau}^{\lambda, i}=X_{-\lambda^{-2} \tau}^{\lambda} W_{\lambda^{-2} \tau}^{\lambda}$, one is led to

$$
W_{\tau}^{\lambda, i}=1+\int_{0}^{\tau} \mathrm{d} \sigma X_{-\lambda-2}^{\lambda} K(\lambda, \tau-\sigma) X_{\lambda^{-2} \sigma}^{\lambda} W_{\sigma}^{\lambda, i},
$$

where the slowly varying kernel $K(\lambda, \tau)$ converges in the weak-coupling limit $\lambda \rightarrow 0$ (under suitable hypotheses) to the celebrated Davies' generator 


$$
K_{\mathrm{D}}=\int_{0}^{\infty} U_{-x} A_{01} U_{x} A_{10} \mathrm{~d} x .
$$

\section{A Family of New Generators}

In our first theorem, we shall find a new class of generators for the semigroup approximation in the van Hove limit. To this purpose, note that $K_{\mathrm{D}}$ has been defined thanks to the change of variable

$$
\left(\begin{array}{l}
\sigma \\
x
\end{array}\right)=\left(\begin{array}{cc}
0 & \lambda^{2} \\
1 & -1
\end{array}\right)\left(\begin{array}{l}
s \\
u
\end{array}\right) .
$$

Here, instead we would like to allow for the most general linear change of variable that keeps a $\lambda^{2}$ jacobian, proper of a second-order approximation, while fixing the relative variable to be $s-u=x$. Accordingly, we take

$$
\left(\begin{array}{c}
\sigma \\
x
\end{array}\right)=\left(\begin{array}{cc}
\left.\frac{1}{2}-\alpha\right) \lambda^{2} & \left(\frac{1}{2}+\alpha\right) \lambda^{2} \\
1 & -1
\end{array}\right)\left(\begin{array}{c}
s \\
u
\end{array}\right)+\left(\begin{array}{c}
\lambda^{2} q \\
0
\end{array}\right)
$$

for some real $\alpha$ and $q$. Some straightforward algebra shows that the integration domain $s=0 \ldots \lambda^{-2} \tau, u=0 \ldots s$ in (1) becomes the domain $\mathcal{D}(\lambda, \tau, \alpha, q)$ in the $(\sigma, x)$ plane given by the triangle of vertices

$$
\mathcal{D}(\lambda, \tau, \alpha, q)=\triangle\left\{\left(\lambda^{2} q, 0\right),\left(\tau+\lambda^{2} q, 0\right),\left(\left(\frac{1}{2}-\alpha\right) \tau+\lambda^{2} q, \lambda^{-2} \tau\right)\right\}
$$

in Fig. 1. Accordingly, (1), written for $W_{\tau}^{\lambda, i}=X_{-\lambda^{-2} \tau} W_{\lambda^{-2} \tau}^{\lambda}$, becomes

$$
\begin{aligned}
W_{\tau}^{\lambda, i}= & 1+\iint_{\mathcal{D}(\lambda, \tau, \alpha, q)} \mathrm{d} \sigma \mathrm{d} x X_{-\lambda^{-2} \sigma+q-\left(\alpha+\frac{1}{2}\right) x}^{\lambda} A_{01} U_{x}^{\lambda} A_{10} X_{\lambda^{-2} \sigma-q+\left(\alpha-\frac{1}{2}\right) x}^{\lambda} \\
& \times W_{\sigma-\lambda^{2}(q+(1 / 2-\alpha) x)}^{\lambda, i} .
\end{aligned}
$$

We now consider the following facts, which will be made precise in Theorem 3.4:

- $\mathcal{D}(\lambda, \tau, \alpha, q) \rightarrow[0, \tau] \times[0, \infty)$ as $\lambda \rightarrow 0$, for any real $\alpha$ and $q$. This justifies the approximation

$$
\iint_{\mathcal{D}(\lambda, \tau, \alpha, q)} \mathrm{d} \sigma \mathrm{d} x \approx \int_{0}^{\tau} \mathrm{d} \sigma \int_{0}^{\infty} \mathrm{d} x \mathrm{e}^{-\frac{(x / 2)^{2}}{2 T(\lambda)^{2}}} \quad \lambda \approx 0,
$$

for some real positive function $T$ of the coupling constant, provided $\lim _{\lambda \rightarrow 0} T(\lambda)=+\infty$. The choice of the gaussian is dictated by later purposes.

- In the weak-coupling limit $\lambda \rightarrow 0$ one could approximate

$$
W_{\sigma-\lambda^{2}(q+(1 / 2-\alpha) x)}^{\lambda, i} \approx W_{\sigma}^{\lambda, i}
$$

in the integral kernel of $(7)$, provided that $T(\lambda)$ does not grow too fast. 


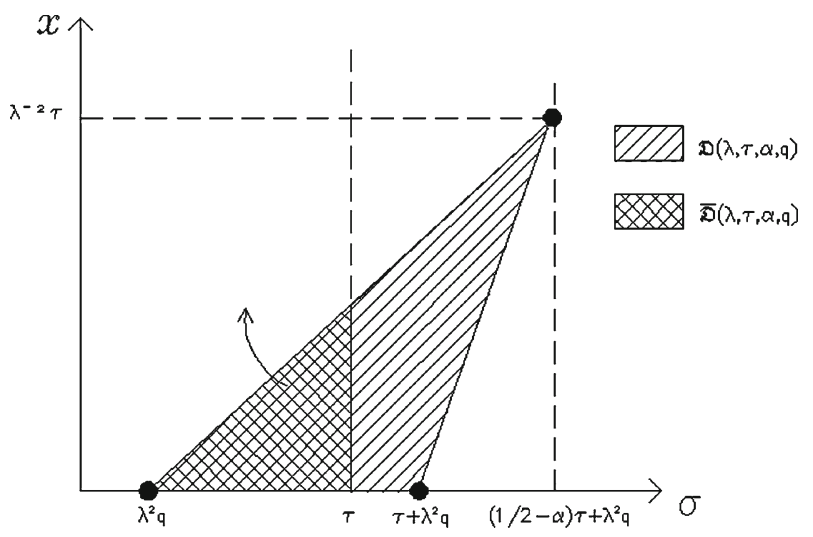

FiguRE 1. Integration domains $\mathcal{D}(\lambda, \tau, \alpha, q)$ and $\overline{\mathcal{D}}(\lambda, \tau, \alpha, q)$ defined in (6) and (33), respectively (we have put $q>0$, $\alpha<-1 / 2$ for clarity). The arrow indicates the asymptotic behavior of the two domains as $\lambda \rightarrow 0$

Then (7) becomes

$$
W_{\tau}^{\lambda, i} \approx 1+\int_{0}^{\tau} \mathrm{d} \sigma X_{-\lambda^{-2} \sigma}^{\lambda} K_{(\alpha, q, T(\lambda))} X_{\lambda^{-2} \sigma}^{\lambda} W_{\sigma}^{\lambda, i}
$$

or, which is the same, $W_{t}^{\lambda} \approx \bar{W}_{t}^{\lambda}$, where we give the following.

Definition 3.1. Let $\alpha, q \in \mathbb{R}$, and $T: \dot{I} \rightarrow \mathbb{R}^{+}$a real positive continuous function on $\dot{I}=[-1,1] \backslash\{0\} \subset \mathbb{R}$. For $\lambda \in \dot{I}$ define the linear operator $K_{(\alpha, q, T(\lambda))}$ on $\mathcal{B}_{0}$ as

$$
K_{(\alpha, q, T(\lambda))}=\int_{0}^{\infty} \mathrm{d} x \mathrm{e}^{-\frac{(x / 2)^{2}}{2 T(\lambda)^{2}}} U_{-\left(\alpha+\frac{1}{2}\right) x+q} A_{01} U_{x} A_{10} U_{\left(\alpha-\frac{1}{2}\right) x-q} .
$$

Denote also with

$$
\bar{W}_{t}^{\lambda}=\exp \left\{\left(Z_{0}+\lambda A_{00}+\lambda^{2} K_{(\alpha, q, T(\lambda))}\right) t\right\}
$$

the associated semigroup on $\mathcal{B}_{0}$.

This is indeed the case, under the same kind of assumptions made in [3], namely

Assumption 3.2. There exists some $0<c<\infty$ such that for every $\bar{\tau}>0$

$$
\int_{0}^{\lambda^{-2} \bar{\tau}}\left\|A_{01} U_{x}^{\lambda} A_{10}\right\| \mathrm{d} x \leq c
$$

is bounded uniformly on $|\lambda| \leq 1$. 
Assumption 3.3. For every $0<\bar{\tau}<\infty$

$$
\lim _{\lambda \rightarrow 0} \int_{0}^{\lambda^{-2} \bar{\tau}}\left\|A_{01}\left(U_{x}^{\lambda}-U_{x}\right) A_{10}\right\| \mathrm{d} x=0 .
$$

Sufficient conditions for these assumptions to hold are given in Sect. 4.3. We denote here and in the sequel with $\dot{I}$ the dotted interval $[-1,1] \backslash\{0\} \subset \mathbb{R}$, and we state our first result:

Theorem 3.4. Suppose that $X_{t}^{\lambda}$ is a one-parameter group of isometries. Let $T: \dot{I} \rightarrow \mathbb{R}^{+}$a real positive continuous function, assume

$$
T(\lambda) \sim|\lambda|^{-\xi} \widetilde{T}, \quad \lambda \rightarrow 0
$$

for some $\widetilde{T}>0$ and $0<\xi<2$ (strictly), and suppose that Assumptions 3.2 and 3.3 hold.

Then for every $\bar{\tau}>0$

$$
\lim _{\lambda \rightarrow 0}\left\{\sup _{0 \leq t \leq \lambda^{-2} \bar{\tau}}\left\|W_{t}^{\lambda}-\bar{W}_{t}^{\lambda}\right\|\right\}=0 .
$$

We defer the proof to Sect. 8, by just commenting that the same philosophy of the proof in [3] is followed, the main difference being the delicate step sketched in (8), which requires a telescopic expansion and is controlled by our bound on $T(\lambda)$. At this point a few comments are of order:

- The dynamical time $T(\lambda)$ (named "dynamical" as it scales with the coupling constant) proves to be an essential, new and natural ingredient of the theory, whose necessity becomes evident as soon as one departs from Davies choices $\alpha=1 / 2, q=0$. With respect to this, the theorem generalizes [3].

- For each choice of $\alpha, q, T$ and nonzero $\lambda$, the corresponding generator $Z_{0}+$ $\lambda A_{00}+K_{(\alpha, q, T(\lambda))}$ is always well defined, irrespective of the spectrum of $Z_{0}$, and self-interactions $A_{00}$ are taken into account.

However, the analysis in [12] shows that Davies generator $K_{\mathrm{D}}=K_{(1 / 2,0,+\infty)}$ need not be dissipative, and it is not evident a priori that any of the $K_{(\alpha, q, T(\lambda))}$ we have found could prove to be dissipative. However, this new class of generators is sufficiently ample to allow for a new type of averaging procedure, close to the idea of temporal averaging introduced in [2], which solves the problem.

\section{Dynamical Time Average}

The idea is to average among $\left\{K_{(\alpha, q, T(\lambda))}\right\}_{q \in \mathbb{R}}$ in the $\alpha$-fibration, using a gaussian probability distribution, whose standard deviation is provided by the natural time scale $T(\lambda)$. The latter goes to infinity in the van Hove limit, but furnishes a well-defined bounded generator for all nonzero $\lambda \mathrm{s}$, irrespective of spectral conditions on $Z_{0}$. 
We start from the simplest case $\alpha=0$, but we will see later on that the same averaging procedure can be applied for any real $\alpha$, always leading to the same, $\alpha$-independent, averaged generator. We give the following.

Definition 4.1. For any real positive $T>0$ put

$$
K_{T}:=\frac{1}{\sqrt{2 \pi} T} \int_{-\infty}^{\infty} \mathrm{d} q \mathrm{e}^{-\frac{q^{2}}{2 T^{2}}} K_{(0, q, T)}=\frac{1}{\sqrt{2 \pi} T} \int_{-\infty}^{\infty} \mathrm{d} q \mathrm{e}^{-\frac{q^{2}}{2 T^{2}}} U_{-q} K_{(0,0, T)} U_{q}
$$

Then, we can prove a markovian approximation theorem for $K_{T(\lambda)}$ under the same conditions of Theorem 3.4, and this constitutes our first main result:

Theorem 4.2. Suppose that $X_{t}^{\lambda}$ is a one-parameter group of isometries. Let $T: \dot{I} \rightarrow \mathbb{R}^{+}$be a real positive continuous function such that

$$
T(\lambda) \sim|\lambda|^{-\xi} \widetilde{T}, \quad \lambda \sim 0
$$

for some $\widetilde{T}>0$ and $0<\xi<2$ (strictly), and denote with

$$
\widetilde{W}_{t}^{\lambda}=\exp \left\{\left(Z_{0}+\lambda A_{00}+\lambda^{2} K_{T(\lambda)}\right) t\right\}
$$

the associated semigroup on $\mathcal{B}_{0}$. Make also Assumptions 3.2 and 3.3.

Then for every $\bar{\tau}>0$

$$
\lim _{\lambda \rightarrow 0}\left\{\sup _{0 \leq t \leq \lambda^{-2} \bar{\tau}}\left\|W_{t}^{\lambda}-\widetilde{W}_{t}^{\lambda}\right\|\right\}=0 .
$$

Again we defer the proof to Sect. 8, while just mentioning that the most delicate point is perhaps the control of uniform bounds on the $q$ variable in the telescopic expansion. It turns out however that integration over $q$ does not pose any problem, as the integration domain scales with $T(\lambda)$ (hence with $\xi$ ), while the various Volterra integral operators involved in the proof of Theorem 3.4 approach each other either uniformly in $q$ or with velocity $2>\xi$. It is worth here to emphasize once again that no specific spectral conditions on $Z_{0}$ are assumed and self-interactions $A_{00}$ are taken into account.

\subsection{Case of Discrete Spectrum}

We make contact with the definition of the time average proposed in [2].

Proposition 4.3. Let $\mathcal{B}_{0}$ be finite dimensional, and $A_{00}=0$. Then for $b \in \mathcal{B}_{0}$ and every $\tau>0$

$$
\lim _{\lambda \rightarrow 0}\left\{\sup _{0 \leq t \leq \lambda^{-2} \tau}\left\|\mathrm{e}^{\left(Z_{0}+\lambda^{2} K_{T(\lambda)}\right) t} b-\mathrm{e}^{\left(Z_{0}+\lambda^{2} K_{\mathrm{D}}^{\natural}\right) t} b\right\|\right\}=0
$$

Proof. Following Theorem 1.4 in [3], it is sufficient to show that $\lim _{\lambda \rightarrow 0}\left\|K_{T(\lambda)}-K_{\mathrm{D}}^{\natural}\right\|=0$. To this end, let

$$
Z_{0}=\sum_{\alpha} i \omega_{\alpha} \Pi_{\alpha}
$$


be the spectral decomposition of $Z_{0}$, with all $\omega_{\alpha}$ s distinct and real, and associated projectors $\Pi_{\alpha}$. Noting that $K_{(0, q, T)}=U_{-q} K_{(0,0, T)} U_{q}$, we compute

$$
\begin{aligned}
\lim _{T \rightarrow+\infty} K_{T} & =\lim _{T \rightarrow+\infty} \frac{1}{\sqrt{2 \pi} T} \int_{-\infty}^{\infty} \mathrm{d} q \mathrm{e}^{-\frac{q^{2}}{2 T^{2}}} \sum_{\alpha \beta} \mathrm{e}^{i\left(\omega_{\alpha}-\omega_{\beta}\right) q} \Pi_{\alpha} K_{(0,0,+\infty)} \Pi_{\beta} \\
& =\sum_{\alpha} \Pi_{\alpha} K_{\mathrm{D}} \Pi_{\alpha} \\
& =\lim _{T \rightarrow+\infty} \frac{1}{2 T} \int_{-T}^{T} \mathrm{~d} q U_{-q} K_{\mathrm{D}} U_{q}
\end{aligned}
$$

which clearly shows that the time average in [2] coincides with our dynamical one, in the weak-coupling limit $\lambda \rightarrow 0$, i.e., recalling that $\lim _{\lambda \rightarrow 0} T(\lambda)=+\infty$,

$$
K_{\mathrm{D}}^{\natural}=\lim _{\lambda \rightarrow 0} K_{T(\lambda)}
$$

and the averaging map $\downarrow$ is well defined because $\mathcal{B}_{0}$ is finite dimensional.

The statement of the proposition is unchanged by only assuming that $Z_{0}$ has discrete spectrum. More importantly, $K_{\mathrm{D}}^{\natural}$ can be recovered as a particular (limit) case of our $K_{T(\lambda)}$. Indeed, by choosing $T(\lambda)=|\lambda|^{-\xi} \bar{\tau}$, for some $0<\xi<2$ and $\bar{\tau}>0$, one has

$$
\lim _{\bar{\tau} \rightarrow+\infty} K_{T(\lambda)}=K_{\mathrm{D}}^{\natural} .
$$

where the limit exists for discrete $Z_{0}$ spectrum due to the computation in the proof above.

\subsection{Structure of $K_{T}$ for Nonzero $\alpha$}

We denote $A(t)=U_{-t} A U_{t}$, and introduce ${ }^{1}$

$$
\delta_{T}(t)=\frac{1}{\sqrt{2 \pi} T} \mathrm{e}^{-\frac{t^{2}}{2 T^{2}}} .
$$

Then according to the change of variable $t_{1}=q+x / 2, t_{2}=q-x / 2$, and because of the properties of the gaussian, $K_{T}$ can be factorized in the following form:

$$
K_{T}=\int_{-\infty}^{\infty} \mathrm{d} t_{1} \sqrt{\delta_{T}}\left(t_{1}\right) A_{01}\left(t_{1}\right) \int_{-\infty}^{t_{1}} \mathrm{~d} t_{2} \sqrt{\delta_{T}}\left(t_{2}\right) A_{10}\left(t_{2}\right) .
$$

We wish to show here that there is nothing peculiar in the choice $\alpha=0$ that leads to the above factorized structure for $K_{T}$, apart from simplicity in the definitions and in the proofs involved. In fact, one could equally well proceed along the following lines: in (1), change variable according to (5) as in Theorem 3.4. Proceed as in the proof of Theorem 3.4, but use $\exp \left\{-\left(t_{1}(x, q)^{2}+t_{2}(x, q)^{2}-\right.\right.$

\footnotetext{
1 This notation for the gaussian is motivated by the fact that the Fourier transform $\sqrt{\delta_{\bar{\omega}}}$ of $\sqrt{\delta_{T}}$, with $\bar{\omega}=1 /(2 T)$, defines a nascent delta function $\delta_{\bar{\omega}}$ in the van Hove limit $\lambda \rightarrow 0$.
} 
$\left.\left.2 q^{2}\right) /\left(4 T(\lambda)^{2}\right)\right\}$ instead of $\exp \left\{-(x / 2)^{2} /\left(2 T(\lambda)^{2}\right)\right\}$ as gaussian smoothing for the kernel in (52), where

$$
\left\{\begin{array}{l}
t_{1}(x, q)=q+(\alpha+1 / 2) x \\
t_{2}(x, q)=q+(\alpha-1 / 2) x
\end{array} .\right.
$$

Then, one can proceed as in the proof Theorem 3.4 to show that (under the same hypotheses) the semigroup $\widehat{W}_{t}^{\lambda}=\exp \left\{\left(Z_{0}+\lambda A_{00}+\lambda^{2} \widehat{K}_{(\alpha, q, T(\lambda))}\right) t\right\}$ satisfies the same markovian approximation theorem with

$$
\widehat{K}_{(\alpha, q, T(\lambda))}=\int_{0}^{\infty} \mathrm{d} x \mathrm{e}^{-\frac{t_{1}(x, q)^{2}+t_{2}(x, q)^{2}-2 q^{2}}{4 T(\lambda)^{2}}} A_{01}\left(t_{1}(x, q)\right) A_{10}\left(t_{2}(x, q)\right) .
$$

Averaging over $q$ with a normalized gaussian distribution of standard deviation $T(\lambda)$, as in Theorem 4.2, gives exactly the same result as in (11).

\subsection{A Sufficient Condition for the Assumptions}

We provide a sufficient condition for the validity of the hypotheses in Theorems 3.4 and 4.2, which is a slight adaptation of Theorem $1.3 \mathrm{in}$ [3], and is supported by perturbation arguments. First, for $n \in \mathbb{N}$ define the coefficients

$$
a_{n}(t)=\int_{0}^{t} \mathrm{~d} t_{0} \ldots \int_{0}^{t_{n-1}} \mathrm{~d} t_{n}\left\|A_{01} U_{t_{0}-t_{1}} A_{11} U_{t_{1}-t_{2}} A_{11} \ldots A_{11} U_{t_{n}} A_{10}\right\|,
$$

which come from the expansion of $U_{t}^{\lambda}$ in powers of $\lambda$.

Theorem 4.4. Suppose that

$$
\int_{0}^{\infty}\left\|A_{01} U_{t_{0}} A_{10}\right\| \mathrm{d} t_{0}<\infty
$$

Suppose that

$$
a_{n}(t) \leq c_{n}|t|^{n / 2}
$$

for all $t \in \mathbb{R}$ and $n \geq 1$, where the series $\sum_{n=1}^{\infty} c_{n} z^{n}$ has infinite radius of convergence. Suppose also that for some $\epsilon>0, d_{n}$, and all $t \geq 0$

$$
a_{n}(t) \leq d_{n}|t|^{n / 2-\epsilon}
$$

Then, Assumptions 3.2 and 3.3 are satisfied.

Proof. By expanding $U_{x}^{\lambda}$ in a $\lambda$ power series, one obtains

$$
\int_{0}^{\lambda^{-2} \bar{\tau}}\left\|A_{01} U_{x}^{\lambda} A_{10}\right\| \mathrm{d} x \leq \sum_{n=0}^{\infty} \lambda^{n} a_{n}\left(\lambda^{-2} \bar{\tau}\right) \leq a_{0}\left(\lambda^{-2} \bar{\tau}\right)+\sum_{n=1}^{\infty} c_{n}|\bar{\tau}|^{n / 2}
$$

which converges for any $\bar{\tau}$ due to hypothesis (12). Similarly,

$$
\int_{0}^{\lambda^{-2} \bar{\tau}}\left\|A_{01}\left(U_{x}^{\lambda}-U_{x}\right) A_{10}\right\| \mathrm{d} x \leq \sum_{n=1}^{\infty} \lambda^{n} a_{n}\left(\lambda^{-2} \bar{\tau}\right)
$$


For $|\lambda|<1$, the series is dominated by the convergent $\sum_{n=1}^{\infty} c_{n}|\bar{\tau}|^{n / 2}$, and each term of the series is dominated by $d_{n}|\lambda|^{2 \epsilon}|\bar{\tau}|^{n / 2-\epsilon}$, which goes to zero when $\lambda \rightarrow 0$, completing the proof.

\section{A Contraction Semigroup}

As a further useful manipulation of $K_{T}$, we name $\Phi^{T}(t)=\sqrt{\delta_{T}}(t) A(t)$ for short and $\Phi_{i j}^{T}(t)=P_{i} \Phi^{T}(t) P_{j}$. Then by usual algebraic manipulations $K_{T}$ can be cast in the form

$$
\begin{aligned}
K_{T}= & \pi \int_{-\infty}^{+\infty} \frac{\mathrm{d} t_{1}}{\sqrt{2 \pi}} \Phi_{01}\left(t_{1}\right) \int_{-\infty}^{+\infty} \frac{\mathrm{d} t_{2}}{\sqrt{2 \pi}} \Phi_{10}\left(t_{2}\right) \\
& +\pi \int_{-\infty}^{+\infty} \frac{\mathrm{d} t_{1}}{\sqrt{2 \pi}} \int_{-\infty}^{t_{1}} \frac{\mathrm{d} t_{2}}{\sqrt{2 \pi}}\left(\Phi_{01}\left(t_{1}\right) \Phi_{10}\left(t_{2}\right)-\Phi_{01}\left(t_{2}\right) \Phi_{10}\left(t_{1}\right)\right)
\end{aligned}
$$

Now we put $\widetilde{\Phi}^{T}=\Phi^{T}-\Phi_{00}^{T}$,

$$
\mathbb{L}_{T}=\int_{-\infty}^{+\infty} \frac{\mathrm{d} t}{\sqrt{2 \pi}} \widetilde{\Phi}^{T}(t)
$$

and define the operator $\widetilde{K}_{T}$ on $\mathcal{B}$ as

$$
\widetilde{K}_{T}=\pi \mathbb{L}_{T}^{2}+\pi \int_{-\infty}^{+\infty} \frac{\mathrm{d} t_{1}}{\sqrt{2 \pi}} \int_{-\infty}^{t_{1}} \frac{\mathrm{d} t_{2}}{\sqrt{2 \pi}}\left[\widetilde{\Phi}^{T}\left(t_{1}\right), \widetilde{\Phi}^{T}\left(t_{2}\right)\right] .
$$

It follows that

$$
K_{T}=P_{0} \widetilde{K}_{T} P_{0}
$$

Now from expressions (14) and (13) one can already understand why $K_{T}$ generates contractions: ${ }^{2} K_{T}$ is the projection of $\widetilde{K}_{T}$, so it generates contractions if the latter does. Now $\widetilde{K}_{T}$ is made of two parts, the first being the square of a generator of isometries (and thus dissipative), and the second a generator of isometries (being a superposition of commutators of generators of isometries).

It is remarkable that the explicit and symmetric forms above (13) and (14) for $K_{T}=P_{0} \widetilde{K}_{T} P_{0}$ can be achieved only through factorization of integration variables for the two time integrals, and this in turn has been possible due to the choice of the gaussians. If one starts from (13) and goes backward using other non-gaussian weights, integrals over $q$ and $x$ do not factor, forbidding a markovian approximation theorem like Theorem 4.2 for $K_{T}$.

Before stating our main result of this section, we report here without proof, for completeness, the part of the Hille-Yosida Theorem [16] we will be using in this section: most operators we are involved with are bounded, as we are assuming $A$ to be bounded.

\footnotetext{
${ }^{2}$ We recall that a semigroup $\mathcal{T}_{t}$ is a contractive if $\left\|\mathcal{T}_{t}\right\| \leq 1$ for all $t \geq 0$.
} 
Theorem 5.1. Let $S$ be a bounded operator on the Banach space $\mathcal{B}$. Then $S$ is the infinitesimal generator of a contraction semigroup if an only if there exist some $\alpha>0$ such that for every $b \in \mathcal{B}$

$$
\|(1-\alpha S) b\| \geq\|b\| .
$$

We shall also need the following.

Lemma 5.2. Let $A$ and $B$ be bounded generators of one-parameter groups of isometries on a Banach space $\mathcal{B}$. Then $C=[A, B]$ generates a one-parameter group of isometries on $\mathcal{B}$.

Proof. Define

$$
F_{t}=\exp \{A t\} \exp \{B t\} \exp \{-A t\} \exp \{-B t\} \quad t \in \mathbb{R}
$$

Clearly, $F_{t}$ is an isometry for every $t \in \mathbb{R}$. Now for $t \in \mathbb{R} \backslash\{0\}$ define

$$
C_{n}(t)=n^{2} t^{-2}\left(F_{t / n}-1\right) .
$$

Then $C_{n}(t)$ generates a one-parameter group of isometries. To show this we take $\alpha \geq 0, b \in \mathcal{B}$, and prove inequality (15) by computing

$$
\begin{aligned}
\left\|\left(1-\alpha\left(F_{t / n}-1\right)\right) b\right\| & =(1+\alpha)\left\|\left(1-\frac{\alpha}{1+\alpha} F_{t / n}\right) b\right\| \\
& \geq(1+\alpha)\left|\|b\|-\frac{\alpha}{1+\alpha}\left\|F_{t / n} b\right\|\right| \geq\|b\|,
\end{aligned}
$$

as $\left\|F_{t / n} b\right\|=\|b\|$. Since $\left\|C_{n}(t)\right\| \leq 2 n^{2} / t^{2}$ is bounded for all $t>0$, it $C_{n}(t)$ generates a one-parameter semigroup of contractions by the Hille-Yosida Theorem. Now a simple calculation [17] shows that $\lim _{n \rightarrow \infty} C_{n}(t)=C$ uniformly, due to boundedness of $A$ and $B$. Then $C$ generates contractions, as for $t \geq 0$ it follows that for any $n>0$,

$$
\begin{aligned}
\left\|\mathrm{e}^{C t}\right\| & \leq\left\|\mathrm{e}^{C_{n}\left(t^{\prime}\right) t}\right\|+\left\|\mathrm{e}^{C t}-\mathrm{e}^{C_{n}\left(t^{\prime}\right) t}\right\| \\
& \leq 1+t \mathrm{e}^{t\left(2\|C\|+\left\|C-C_{n}\left(t^{\prime}\right)\right\|\right)}\left\|C-C_{n}\left(t^{\prime}\right)\right\|
\end{aligned}
$$

(see Chapter 3.1.1 in [18]). Inverting the role of $A$ and $B$ in all of the above shows that $-C$ also generates contractions, thus proving the Lemma.

We can now state the main result of this section (second main result):

Theorem 5.3. If $\left\|P_{0}\right\|=1$, then $\widetilde{W}_{t}^{\lambda}$ is a contraction semigroup on $\mathcal{B}_{0}$, for all real $\lambda \neq 0$.

Proof. $X_{t}^{\lambda}$ is a one-parameter group of isometries because of Lemma 1.1 in [3]. Now because of the Trotter product formula [19], one has

$$
P_{0} \widetilde{W}_{t}^{\lambda}=P_{0} \mathrm{e}^{\left(Z_{0}+\lambda A_{00}+\lambda^{2} K_{T(\lambda)}\right) t}=\lim _{n \rightarrow \infty}\left\{X_{t / n}^{\lambda} \mathrm{e}^{\lambda^{2} K_{T(\lambda)} t / n}\right\}^{n},
$$

so that

$$
\left\|P_{0} \widetilde{W_{t}^{\lambda}}\right\| \leq \sup _{n}\left\|P_{0} \mathrm{e}^{\lambda^{2} K_{T(\lambda)} t / n}\right\|^{n}
$$

and the theorem would follow if $K_{T}$ would generate a contraction semigroup on $\mathcal{B}_{0}$, for all $T>0$. To show this is indeed the case, we consider its form given by 
(13) with $K_{T}=P_{0} \widetilde{K}_{T} P_{0}$. Now again through the Trotter product formula, the theorem would follow from the fact that $\widetilde{K}_{T}$ generates a contraction semigroup on $\mathcal{B}$. Indeed,

$$
\begin{aligned}
P_{0} \mathrm{e}^{K_{T} t} & =\lim _{n \rightarrow \infty} P_{0}\left\{1+P_{0} \widetilde{K}_{T} P_{0} t / n+O\left(n^{-2}\right)\right\}^{n} \\
& =\lim _{n \rightarrow \infty}\left\{P_{0} \mathrm{e}^{\widetilde{K}_{T} t / n} P_{0}\right\}^{n},
\end{aligned}
$$

so that, since $\left\|P_{0}\right\|=1$, we obtain

$$
\left\|P_{0} \mathrm{e}^{K_{T} t}\right\| \leq\left\|\mathrm{e}^{\widetilde{K}_{T} t}\right\|
$$

for all $t>0$. So we shall prove that $\widetilde{K}_{T}$ generates a contraction semigroup on $\mathcal{B}$ by showing that each of the two terms in (13) does.

In order to do that, we note that $\widetilde{\Phi}^{T}(t)$ generates isometries for all $t$, as for all real $\alpha$ and $b \in \mathcal{B}$ we have

$$
\left\|\left(1-\alpha \Phi^{T}(t)\right) b\right\| \geq\left\|\left(1-\alpha \sqrt{\delta_{T}}(t) A\right) U_{t} b\right\| \geq\|b\|
$$

because $A$ generates isometries and $U_{ \pm t}$ are isometries, and from Trotter formula, and $\left\|P_{0}\right\|=1$ (see above), it follows that $\Phi_{00}^{T}(t)$ generates isometries as well for all $t$.

Then by linearity it follows that $\mathbb{L}_{T}$ (bounded by $\left(\frac{2}{\pi}\right)^{\frac{1}{4}} \sqrt{T}\|A\|$ ) generates isometries, as if $\eta_{b}$ is any tangent functional at $b \in \mathcal{B}$ it follows that $\Re \eta_{b}\left(\int \mathrm{d} t \widetilde{\Phi}^{T}(t) b\right)=\int \mathrm{d} t \Re \eta_{b}\left(\widetilde{\Phi}^{T}(t) b\right)=0$ since $\widetilde{\Phi}^{T}(t)$ is conservative for every $t$ (see e.g. Proposition 3.1.14 in [18]).

It then follows that the bounded $\mathbb{L}_{T}^{2}$ generates a contraction semigroup, as

$$
\left\|\left(1-\alpha \mathbb{L}_{T}^{2}\right) b\right\|=\left\|\left(1-\sqrt{\alpha} \mathbb{L}_{T}\right)\left(1+\sqrt{\alpha} \mathbb{L}_{T}\right) b\right\| \geq\|b\|
$$

for any $\alpha>0$ and $b \in \mathcal{B}$, by repeated use of the Hille-Yosida theorem.

To treat the remaining term in (13), we have already noted that $\widetilde{\Phi}^{T}(t)$ generates isometries for all $t$, so Lemma 5.2 implies that $\left[\widetilde{\Phi}^{T}\left(t_{1}\right), \widetilde{\Phi}^{T}\left(t_{2}\right)\right]$ generates isometries as well. By linearity of the tangent functionals and the fact that

$$
\left\|\int_{-\infty}^{+\infty} \frac{\mathrm{d} t_{1}}{\sqrt{2 \pi}} \int_{-\infty}^{t_{1}} \frac{\mathrm{d} t_{2}}{\sqrt{2 \pi}}\left[\widetilde{\Phi}^{T}\left(t_{1}\right), \widetilde{\Phi}^{T}\left(t_{2}\right)\right]\right\| \leq \frac{2 T}{\sqrt{2 \pi}}\|A\|^{2}
$$

is bounded, it follows that the operator in the norm at the left hand side generates a one-parameter group of isometries. This completes the proof.

\section{Quantum Fokker-Planck Equation}

In this section, we will address the problem of positivity of the contraction semigroup $\widetilde{W_{t}^{\lambda}}$, generated by $Z_{0}+\lambda A_{00}+K_{T(\lambda)}$. In order to give meaning to that, we need some algebraic structure, so we will restrict our attention to the case $\mathcal{B}=\mathcal{A}$ is a $C^{*}$-algebra with identity [20], and $P_{0}$ is a (completely positive) conditional expectation projecting onto the $C^{*}$-subalgebra $\mathcal{X} \hookrightarrow \mathcal{A}$. 
Definition 6.1. For strictly positive $\mathrm{T}$ and self-adjoint $H^{\prime} \in \mathcal{A}$ define the selfadjoint averaged perturbation $\mathcal{L}_{T} \in \mathcal{A}$ as

$$
\mathcal{L}_{T}=\int_{-\infty}^{\infty} \frac{\mathrm{d} t}{\sqrt{2 \pi}} \sqrt{\delta_{T}}(t) U_{t}\left(H^{\prime}\right)
$$

and put $\widetilde{\mathcal{L}}_{T}=P_{1}\left(\mathcal{L}_{T}\right) \in \mathcal{A}$. Moreover, define $\mathcal{G}_{T} \in \mathcal{A}$ through

$$
\mathcal{G}_{T}=\frac{i}{2} \int_{-\infty}^{+\infty} \int_{\sqrt{2 \pi}} \frac{\mathrm{d} t_{1}}{\sqrt{2 \pi}} \frac{\mathrm{d} t_{2}}{\sqrt{2 \pi}} \operatorname{sign}\left(t_{1}-t_{2}\right) \sqrt{\delta_{T}}\left(t_{1}\right) \sqrt{\delta_{T}}\left(t_{2}\right) P_{1}\left(U_{t_{1}}\left(H^{\prime}\right)\right) P_{1}\left(U_{t_{2}}\left(H^{\prime}\right)\right)
$$

We can now state our third main result:

Theorem 6.2. Let $P_{0}$ be a (completely) positive conditional expectation onto a $C^{*}$-subalgebra $\mathcal{X} \hookrightarrow \mathcal{A}$. Let $U_{t}=\exp \{Z t\}$ be a one-parameter group of automorphisms on $\mathcal{A}$. Assume that $\left[Z, P_{0}\right]=0$ and that $Z_{0}$ generates a oneparameter group of automorphisms on $\mathcal{X}$. Suppose also that $A=i\left[H^{\prime}, \cdot\right]$ for some self-adjoint $H^{\prime} \in \mathcal{A}$. Then

(i) $X_{t}^{\lambda}=e^{\left(Z_{0}+\lambda A_{00}\right) t}$ is a one-parameter group of automorphisms on $\mathcal{X}$.

(ii) The contraction semigroup $\widetilde{W}_{t}^{\lambda}=\exp \left\{\left(Z_{0}+\lambda A_{00}+\lambda^{2} K_{T(\lambda)}\right) t\right\}$ on $\mathcal{X}$ is a dynamical semigroup [(completely) positive and identity preserving].

(iii) Its generator on $\mathcal{X}$, that we name "Quantum Fokker-Planck Equation", has the Lindblad form

$$
\begin{aligned}
\partial_{t} X= & Z_{0}(X)+i \lambda\left[P_{0}\left(H^{\prime}\right), X\right]+2 \pi \lambda^{2} i\left[P_{0}\left(\mathcal{G}_{T(\lambda)}\right), X\right] \\
& +2 \pi \lambda^{2}\left(-\frac{1}{2}\left\{\left(P_{0}\left(\widetilde{\mathcal{L}}_{T(\lambda)}\right)\right)^{2}, X\right\}+P_{0}\left(\widetilde{\mathcal{L}}_{T(\lambda)} X \widetilde{\mathcal{L}}_{T(\lambda)}\right)\right)
\end{aligned}
$$

with $P_{0}\left(\mathcal{G}_{T(\lambda)}\right) \in \mathcal{X}$ self-adjoint.

Proof. To prove (i) note that $P_{0}\left(H^{\prime}\right)$ is self-adjoint, as $H^{\prime}$ is self-adjoint and $P_{0}$ is an adjoint map. Then $\left.A_{00}\right|_{\mathcal{X}}=\left.i\left[P_{0}\left(H^{\prime}\right), \cdot\right]\right|_{\mathcal{X}}$ generates a one-parameter group of automorphisms on $\mathcal{X}$, and so does $Z_{0}$ by hypothesis. The validity of (ii) follows from (iii), by just noting that equation (iii) is in the Lindblad form [21]. In fact, both $X \mapsto P_{0}(X)$ and $X \mapsto \widetilde{\mathcal{L}}_{\lambda} X \widetilde{\mathcal{L}}_{\lambda}$ are completely positive maps (the latter is completely positive since it has the Kraus form [22]), and so is their composition. Moreover $\mathcal{G}$, and so $P_{0}(\mathcal{G})$, is evidently self-adjoint. The remaining requirement $\partial_{t} 1_{\mathcal{X}}=0$ of identity preservation can be easily checked upon proving (iii).

To show (iii), we consider $K_{T(\lambda)}=P_{0} \widetilde{K}_{T(\lambda)} P_{0}$ with $\widetilde{K}_{T(\lambda)}$ defined in (13). In order to compute $K_{T(\lambda)}$, let us also note that for every $X, Y \in \mathcal{A}$, and real $t, U_{t}(X Y)=U_{t}(X) U_{t}(Y)$ since $U_{t}$ is an automorphism. Then, it follows that

$$
A_{i j}(t)(X)=U_{-t} A_{i j} U_{t}(X)=i P_{i}\left(\left[U_{-t}\left(H^{\prime}\right), P_{j}(X)\right]\right) .
$$


We will now separately consider each of the two terms of $K_{T}=P_{0} \widetilde{K}_{T} P_{0}$, for $\widetilde{K}_{T}$ in (13). Since for a generic $X \in \mathcal{A}$ we compute

$$
\begin{aligned}
\int_{-\infty}^{+\infty} \frac{\mathrm{d} t}{\sqrt{2 \pi}} \widetilde{\Phi}^{T}(t)(X) & =i\left[\mathcal{L}_{T}, X\right]-i P_{0}\left(\left[\mathcal{L}_{T}, P_{0}(X)\right]\right) \\
& =i\left[P_{1}\left(\mathcal{L}_{T}\right), X\right]+i\left[P_{0}\left(\mathcal{L}_{T}\right), P_{1}(X)\right]
\end{aligned}
$$

because $P_{0}$ is a conditional expectation, so $P_{0}\left(Y \mathcal{L}_{T} Z\right)=Y P_{0}\left(\mathcal{L}_{T}\right) Z$ for $Y, Z \in$ $\mathcal{X}$. We take some $X=P_{0}(X) \in \mathcal{X}$ (in which case the second term at the right hand side in the equation above disappears), and using again the latter property we compute

$$
\begin{aligned}
P_{0} \mathbb{L}_{T}^{2} P_{0}(X) & =-P_{0}\left(\left[P_{1}\left(\mathcal{L}_{T}\right),\left[P_{1}\left(\mathcal{L}_{T}\right), X\right]\right]\right)-P_{0}\left(\left[P_{0}\left(\mathcal{L}_{T}\right), P_{1}\left(\left[P_{1}\left(\mathcal{L}_{T}\right), X\right]\right)\right]\right) \\
& =-\left\{P_{0}\left(\left(P_{1}\left(\mathcal{L}_{T}\right)\right)^{2}\right), X\right\}+2 P_{0}\left(P_{1}\left(\mathcal{L}_{T}\right) X P_{1}\left(\mathcal{L}_{T}\right)\right),
\end{aligned}
$$

since $P_{0} P_{1}=0$. The second term at the right hand side of (13), applied to some $X \in \mathcal{X}$, and projected, can be treated with in the same way: we denote $H_{t}^{\prime}=U_{t}\left(H^{\prime}\right), \widetilde{A}(t)=A(t)-P_{0} A(t) P_{0}$ where as before $A(t)=U_{-t} A U_{t}$, and for $X \in \mathcal{X}$ we compute

$$
\begin{aligned}
P_{0} \widetilde{A}\left(t_{1}\right) \widetilde{A}\left(t_{2}\right)(X)= & -P_{0}\left(P_{1}\left(H_{t_{1}}^{\prime}\right) P_{1}\left(H_{t_{2}}^{\prime}\right)\right) X-X P_{0}\left(P_{1}\left(H_{t_{2}}^{\prime}\right) P_{1}\left(H_{t_{1}}^{\prime}\right)\right) \\
& +P_{0}\left(P_{1}\left(H_{t_{1}}^{\prime}\right) X P_{1}\left(H_{t_{2}}^{\prime}\right)\right)+P_{0}\left(P_{1}\left(H_{t_{2}}^{\prime}\right) X P_{1}\left(H_{t_{1}}^{\prime}\right)\right)
\end{aligned}
$$

Multiplying by $\frac{1}{2} \sqrt{\delta_{T}}\left(t_{1}\right) \sqrt{\delta_{T}}\left(t_{2}\right) \operatorname{sign}\left(t_{1}-t_{2}\right)$ and integrating on all space $\left(t_{1}, t_{2}\right) \in \mathbb{R}^{2}$ gives the projection $P_{0}$ of the second term at the right hand side of (13), applied to any $X \in \mathcal{X}$. Due to antisymmetry of the sign function the second line above disappears, so that

$$
\begin{aligned}
\pi & P_{0} \iint_{\mathbb{R}^{2}} \frac{\mathrm{d} t_{1}}{\sqrt{2 \pi}} \frac{\mathrm{d} t_{2}}{\sqrt{2 \pi}} \operatorname{sign}\left(t_{1}-t_{2}\right) \widetilde{\Phi}^{T}\left(t_{1}\right) \widetilde{\Phi}^{T}\left(t_{2}\right)(X) \\
= & -\pi \iint_{\mathbb{R}^{2}} \frac{\mathrm{d} t_{1}}{\sqrt{2 \pi}} \frac{\mathrm{d} t_{2}}{\sqrt{2 \pi}} \sqrt{\delta_{T}}\left(t_{1}\right) \sqrt{\delta_{T}}\left(t_{2}\right) \\
& \times P_{0}\left(\left[P_{1}\left(H_{t_{1}}^{\prime}\right), P_{1}\left(H_{t_{2}}^{\prime}\right)\right]\right) X+X P_{0}\left(\left[P_{1}\left(H_{t_{2}}^{\prime}\right), P_{1}\left(H_{t_{1}}^{\prime}\right)\right]\right)
\end{aligned}
$$

Standard manipulation on the integration domain shows that this is equal to $2 \pi i\left[P_{0}\left(\mathcal{G}_{T}\right), X\right]$, and the proof is concluded by noting that $P_{1}$ and $P_{0}$ are adjoint maps, so that $P_{0}\left(\mathcal{G}_{T}\right)$ is (bounded and) self-adjoint.

Corollary 6.3. If $\mathcal{A}$ is a $W^{*}$-algebra with identity, $\mathcal{X}$ is a $W^{*}$-subalgebra, and $P_{0}$ is a normal completely positive conditional expectation, then under the hypotheses of the last theorem $\widetilde{W}_{t}^{\lambda}$ is a Quantum Dynamical Semigroup $(Q D S)$ in the sense of [21].

\section{Comments:}

- The dynamical system above is always well defined irrespective of spectral conditions on $Z_{0}$, and even when $\operatorname{sp}\left(Z_{0}\right)$ is discrete, it generalizes literature in that first-order terms $P_{0}\left(H^{\prime}\right)$ need not vanish. 
- If $P_{0}$ is completely positive, then $\left\|P_{0}\right\|=\left\|P_{0}(1)\right\|=1$ (see e.g. [21]), in agreement with the hypotheses of the validity of Theorem 5.3.

- $\quad P_{0}$ need not be the partial trace over a heat bath [13].

- Put in the form of the above theorem, $K_{T(\lambda)}$ furnishes a dynamical measure of the obstruction for $P_{0}$ to be an algebra homomorphism (as $K_{T(\lambda)}=0$ in that case), thus giving dynamical information on the physical subsystem $\mathcal{X} \hookrightarrow \mathcal{A}$.

\section{Example: A Free Particle Coupled to a Heat Bath}

We would like to discuss here the limit dynamics for the prototypical example in Quantum Open System, that of the partial trace on a bipartite system, in case $Z_{0}$ has purely continuous spectrum. The situation can be easily generalized to more reservoirs and/or to different forms for the coupling hamiltonian. However, it turns out that the number of different possibilities is considerably larger with respect to the discrete case, and in order to address steadiness we shall eventually choose to focus on the concrete case of a free nonrelativistic point particle, whose interaction with a fermionic heat bath depends on its energy transitions only.

Although the (physically realistic) example is chosen for its simplicity, it is clear from the analysis that the same conclusions would apply to more general situations (spin degrees of freedom could easily be dealt with, some potential profiles may be included, etc.): because of this reason, we shall now start our analysis at a fairly general level, and only at a second stage shall we specialize to our free particle and our heat bath.

Let $\mathcal{H}=\mathcal{H}_{A} \otimes \mathcal{H}_{B}$ be the tensor product of two Hilbert spaces and $\sigma=|\Omega\rangle\langle\Omega|$ a faithful normal state in $\mathcal{T}\left(\mathcal{H}_{B}\right)(\mathcal{T}$ stands for trace-class operators). Define the normal conditional expectation in the $W^{*}$-algebra $\mathcal{A}=\mathcal{B}(\mathcal{H})$ ( $\mathcal{B}$ stands for bounded operators) according to

$$
P_{0}(X \otimes Y)=\langle Y\rangle_{\sigma} X \otimes 1
$$

and extension by linearity, $\langle Y\rangle_{\sigma}$ being the expectation of $Y$ on the state $\sigma$. The range of $P_{0}$ is identified with the $W^{*}$-subalgebra $\mathcal{X}=\mathcal{B}\left(\mathcal{H}_{A}\right)$.

To discuss the dynamics, we suppose that $\tau_{t}=\mathrm{e}^{i\left[H_{A}, \cdot\right] t}$ is a weakly continuous one-parameter group of automorphisms of the $W^{*}$-dynamical system $(\tau, \mathcal{X})$, for an (unbounded) self-adjoint hamiltonian $H_{A}$. We let the same hold for the $W^{*}$-dynamical system $\left(\mathrm{e}^{i\left[H_{B}, \cdot\right] t}, \mathcal{B}\left(\mathcal{H}_{B}\right)\right)$, and suppose that $\sigma$ is invariant under the latter, so that $\left[Z, P_{0}\right]=0$. Now consider the self-adjoint hamiltonian on $\mathcal{H}$, of the form

$$
H_{\lambda}=H_{A} \otimes 1+1 \otimes H_{B}+\lambda H_{I}
$$

with interaction

$$
H_{I}=Q \otimes \Phi
$$


where $Q$ and $\Phi$ are (bounded) self-adjoint operators on the respective spaces. Name the connected bath continuous correlation function as

$$
h(t)=\left\langle\mathrm{e}^{i H_{B} t}\left(\Phi-\langle\Phi\rangle_{\sigma}\right) \mathrm{e}^{-i H_{B} t}\left(\Phi-\langle\Phi\rangle_{\sigma}\right)\right\rangle_{\sigma},
$$

suppose $h$ is integrable and let

$$
\int_{0}^{\infty} \frac{\mathrm{d} t}{\sqrt{2 \pi}} \mathrm{e}^{i \omega t} h(t)=\frac{1}{2} \hat{h}(\omega)+i s(\omega)
$$

We have already shown that (16) in Theorem 6.2 defines a QDS, and it is evident that $K_{T}$ itself generates a norm-continuous (quantum) dynamical semigroup if $P_{0}$ is the partial trace. However, to gain insight in the study of equilibrium states, an energy spectral resolution is imperative. To do that, we shall already at this point limit our analysis to subsystems for which the following assumption is valid:

Assumption 7.1. There exist bounded $A_{\omega} \in \mathcal{X}, \omega \in \mathbb{R}$ such that $\omega \mapsto\left\|A_{\omega}\right\|$ is integrable and the following expansion

$$
\tau_{t}(Q)=\int \mathrm{d} \omega \mathrm{e}^{-i \omega t} A_{\omega}
$$

holds true. Moreover $\tau_{t}\left(A_{\omega}\right)=\mathrm{e}^{-i \omega t} A_{\omega}$ for every $t \in \mathbb{R}$.

It is reasonable to expect that the last condition should in some way be implied by the group property of $\tau_{t}$, but this is not completely evident a priori, at least in the general case. Note that Assumption 7.1 can only hold if $H_{A}$ has purely continuous spectrum.

Upon identifying $X \sim X \otimes 1$ for $X \in B\left(\mathcal{H}_{A}\right)$ we compute from (16) that $Z_{0}(X)=i\left[H_{A}, X\right]$ and $A_{00}(X)=i\langle\Phi\rangle_{\sigma}[Q, X]$. Then we give the following

Proposition 7.2. For $\bar{\omega}=(2 T)^{-1}>0$ and under Assumption 7.1,

$$
\begin{aligned}
K_{T}(X)= & -2 \pi i \int \frac{\mathrm{d} \omega}{\sqrt{2 \pi}} s(\omega)\left[A_{\omega, \bar{\omega}}^{\dagger} A_{\omega, \bar{\omega}}, X\right] \\
& +2 \pi \int \frac{\mathrm{d} \omega}{\sqrt{2 \pi}} \hat{h}(\omega)\left(-\frac{1}{2}\left\{A_{\omega, \bar{\omega}}^{\dagger} A_{\omega, \bar{\omega}}, X\right\}+A_{\omega, \bar{\omega}}^{\dagger} X A_{\omega, \bar{\omega}}\right)
\end{aligned}
$$

is norm bounded, with bounded

$$
A_{\omega, \bar{\omega}}=\int \mathrm{d} \omega^{\prime} \sqrt{\delta_{\bar{\omega}}}\left(\omega-\omega^{\prime}\right) A_{\omega^{\prime}}
$$

Proof. We shall only treat the term $P_{0}\left(\mathcal{G}_{T}\right)$ in (16), all the others being completely analogous. To this purpose, note that

$$
P_{1}\left(U_{t}\left(H^{\prime}\right)\right)=\tau_{t}(Q) \otimes \mathrm{e}^{i H_{B} t}\left(\Phi-\langle\Phi\rangle_{\sigma}\right) \mathrm{e}^{-i H_{B} t},
$$

so that

$$
\mathcal{G}_{T}=\frac{i}{2} \iint_{-\infty}^{+\infty} \frac{\mathrm{d} t_{1}}{\sqrt{2 \pi}} \frac{\mathrm{d} t_{2}}{\sqrt{2 \pi}} h\left(t_{1}-t_{2}\right) \operatorname{sign}\left(t_{1}-t_{2}\right) \sqrt{\delta_{T}}\left(t_{1}\right) \sqrt{\delta_{T}}\left(t_{2}\right) \tau_{t_{1}}(Q) \tau_{t_{2}}(Q)
$$


Then we pass to $t_{1,2}=q \pm r / 2$ and use Assumption 7.1 above to compute

$$
\begin{aligned}
P_{0}\left(\mathcal{G}_{T}\right)= & \pi i \iint \mathrm{d} \omega_{\alpha} \mathrm{d} \omega_{\beta} \int \frac{\mathrm{d} q}{\sqrt{2 \pi}} \mathrm{e}^{-i\left(\omega_{\alpha}+\omega_{\beta}\right) q} \sqrt{\delta_{T}}(\sqrt{2} q) \\
& \times \int \frac{\mathrm{d} r}{\sqrt{2 \pi}} \mathrm{e}^{-i\left(\frac{\omega_{\alpha}-\omega_{\beta}}{2}\right) r} \sqrt{\delta_{T}}(r / \sqrt{2}) h(r) \operatorname{sign}(r) A_{\omega_{\alpha}} A_{\omega_{\beta}}
\end{aligned}
$$

because $\sqrt{\delta_{T}}\left(t_{1}\right) \sqrt{\delta_{T}}\left(t_{2}\right)=\sqrt{\delta_{T}}(\sqrt{2} q) \sqrt{\delta_{T}}(r / \sqrt{2})$. Now the integral over $q$ is just $2^{-1 / 2} \sqrt{\delta_{\bar{\omega}}}\left(\left(\omega_{\alpha}+\omega_{\beta}\right) / \sqrt{2}\right)$ and Fourier transform for $\sqrt{\delta_{T}}(r / \sqrt{2})$ gives

$$
\mathrm{e}^{-i\left(\frac{\omega_{\alpha}-\omega_{\beta}}{2}\right) r} \sqrt{\delta_{T}}(r / \sqrt{2})=\sqrt{2} \int \frac{\mathrm{d} \omega}{\sqrt{2 \pi}} \mathrm{e}^{i \omega r} \sqrt{\delta_{\bar{\omega}}}\left(\sqrt{2} \omega+\frac{\omega_{\alpha}-\omega_{\beta}}{\sqrt{2}}\right)
$$

Because of the properties of the gaussian we also have

$$
\sqrt{\delta_{\bar{\omega}}}\left(\omega+\omega_{\alpha}\right) \sqrt{\delta_{\bar{\omega}}}\left(\omega-\omega_{\beta}\right)=\sqrt{\delta_{\bar{\omega}}}\left(\frac{\omega_{\alpha}+\omega_{\beta}}{\sqrt{2}}\right) \sqrt{\delta_{\bar{\omega}}}\left(\sqrt{2} \omega+\frac{\omega_{\alpha}-\omega_{\beta}}{\sqrt{2}}\right)
$$

so that

$$
P_{0}\left(\mathcal{G}_{T}\right)=2 \pi \int \frac{\mathrm{d} \omega}{\sqrt{2 \pi}}\left(\frac{i}{2} \int \frac{\mathrm{d} r}{\sqrt{2 \pi}} \mathrm{e}^{i \omega r} h(r) \operatorname{sign}(r)\right) A_{-\omega, \bar{\omega}} A_{\omega, \bar{\omega}} .
$$

The term inside the parentheses is just $-s(\omega)$, and observing that $A_{-\omega, \bar{\omega}}=$ $A_{\omega, \bar{\omega}}^{\dagger}$ gives the form of $P_{0}\left(\mathcal{G}_{T}\right)$ stated in the proposition. As said, the other terms all follow in the same way, and $K_{T}(X)$ written in terms of $A_{\omega, \bar{\omega}}$ is norm bounded as $(\bar{h}(\omega)+i s(\omega))\left\|A_{\omega, \bar{\omega}}\right\|^{2}$ is integrable.

Note that the presence of first-order contributions manifests itself in that, with physical and diagrammatical terminology, only "connected" correlation functions $h(t)$ appear to second order. Note also that the spectral theorem for $H_{B}$ reveals $\hat{h}(\omega) \geq 0$ for all real $\omega$.

The assumption of boundedness for the $\left\|A_{\omega}\right\|$ in (17), and even of their existence, can surely be relaxed, as no mention to $A_{\omega}$ or restriction on the spectrum of $Z_{0}$ is made in the following

Proposition 7.3. The form of $K_{T}$ in Proposition 7.2 follows if

1. $h(t)$ is integrable and $h(0)=0$

2. $\hat{h}(\omega)$ and $s(\omega)$ are integrable

by defining

$$
A_{\omega, \bar{\omega}}=\int \frac{\mathrm{d} t}{\sqrt{2 \pi}} \mathrm{e}^{i \omega t} \sqrt{\delta_{T}}(t) \tau_{t}(Q) .
$$

Then $A_{\omega, \bar{\omega}}$ is bounded uniformly on $\omega \in \mathbb{R}$ and $K_{T}$ is norm bounded.

Proof. We have

$$
h(t) \operatorname{sign}(t)=\int \frac{\mathrm{d} \omega}{\sqrt{2 \pi}} \mathrm{e}^{-i \omega t} s(\omega)
$$

because $h(t) \operatorname{sign}(t)$ admits Fourier transform (as $h(t)$ is integrable by hypothesis), vanishes at infinity and is uniformly continuous (as $h(t)$ is the inverse 
Fourier transform of $\hat{h}(\omega)$ which is integrable by hypothesis and $h(0)=0)$. By plugging this into $(20)$ we immediately obtain $P_{0}(\mathcal{G})$ as in Proposition 7.2 , with $A_{\omega, \bar{\omega}}$ defined above, and the other terms follow analogously (with no requirement on $h(0))$. Then $\left\|A_{\omega, \bar{\omega}}\right\| \leq(2 / \pi)^{\frac{1}{4}} \sqrt{T}\|Q\|$ is bounded uniformly on $\omega$ and $K_{T}$ is norm bounded because of hypothesis (2).

As a brief comment, the technical hypothesis $h(0)=0$ avoids energy renormalization singularities in the time domain, corresponds to $\left\langle\Phi^{2}\right\rangle_{\sigma}=\langle\Phi\rangle_{\sigma}^{2}$, and explicitly requires nonzero first-order terms $\left(\langle\Phi\rangle_{\sigma} \neq 0 \Leftrightarrow A_{00} \neq 0\right)$. Despite of this, the hypothesis has nothing to do with the dissipative part of $K_{T}$, as noted in the proof above and, as evident from Proposition 7.2, could surely be relaxed by providing suitable additional information on the subsystem $\mathcal{X}$.

In passing, we have already proven in the general case (Sect. 4.1) that the limit $T \rightarrow \infty$ is well defined and corresponds to Davies averaged $K^{\natural}$, if $\operatorname{sp}\left(Z_{0}\right)$ is discrete and $A_{00}=0$ (which here corresponds to $\langle\Phi\rangle_{\sigma}=0$ ). Here it is possible to see that even when $\langle\Phi\rangle_{\sigma} \neq 0$ one can write precisely Eq. (4.9) in [2] by just substituting the "connected" $h$ and $s$ we have defined. ${ }^{3}$ Indeed, this is found by expanding $\tau_{t}(Q)=\sum_{i \omega \in \operatorname{sp}_{\mathrm{d}}\left(Z_{0}\right)} \widetilde{A}_{\omega} \mathrm{e}^{-i \omega t}$ in $(20)$ : the limit $\bar{\omega} \rightarrow 0$ of the right hand side of $(21)$ gives $\delta_{\omega_{\beta},-\omega_{\alpha}} \delta\left(\omega-\omega_{\alpha}\right)$, where the first is a Kronecker delta, and the second is a Dirac delta.

Reasoning backwards in the frequency domain, it is evident how our new average procedure has smoothed the product of a Kronecker times a Dirac delta, by letting the singularity be shared symmetrically and without restrictions on the spectrum of $Z_{0}$; the gaussian form of our weights then allows to factorize frequencies according to (21), and always because gaussians are involved, the factorization remains even in the time domain, both for $t_{1,2}$ (proving that $K_{T}$ is dissipative) and for $t_{1} \pm t_{2} / 2$ (proving the markovian approximation theorem).

The only side effect is that now the resonance of $A_{\omega, \bar{\omega}}$ has been smoothed to roughly $\omega \pm \bar{\omega}$, so that $K_{T}$ does not preserve the set of diagonal elements $\mathcal{D}=\left\{X \in \mathcal{B}\left(\mathcal{H}_{A}\right) \mid \tau_{t}(X)=X, \forall t \in \mathbb{R}\right\}$. Moreover, for purely continuous $Z_{0}$ spectrum one has $K_{T} \stackrel{T \rightarrow \infty}{\longrightarrow} 0$ strongly, as evident from the above discussion. However, there is still a means to compare with Eq. (4.9) in [2]:

Definition 7.4. Under Assumption 7.1, $h(t)$ integrable and $\omega \mapsto\left\|A_{\omega}\right\|$ square integrable, we define the operator $L$ on $\mathcal{X}$ given by

$$
\begin{aligned}
L(X)= & -2 \pi i \int \mathrm{d} \omega s(\omega)\left[A_{-\omega} A_{\omega}, X\right] \\
& +2 \pi \int \mathrm{d} \omega \hat{h}(\omega)\left(-\frac{1}{2}\left\{A_{-\omega} A_{\omega}, X\right\}+A_{-\omega} X A_{\omega}\right)
\end{aligned}
$$

Proposition 7.5. Suppose $h$ to be integrable. Under Assumption 7.1 suppose that $\mathbb{R} \ni \omega \mapsto A_{\omega} \in \mathcal{X}$ is continuous and

$$
\left\|A_{\omega}\right\|=o\left(|\omega|^{1 / 2}\right), \quad|\omega| \rightarrow \infty
$$

3 The additional factor $\sqrt{2 \pi}$ is due to different definitions of Fourier transform. 
Then $L$ is bounded, generates a norm continuous $Q D S$ on $\mathcal{X}$ and $L$ preserves $\mathcal{D}=\left\{X \in \mathcal{B}\left(\mathcal{H}_{A}\right) \mid \tau_{t}(X)=X, \forall t \in \mathbb{R}\right\}$ in the sense that $e^{L t}(X) \in \mathcal{D}$ whenever $X \in \mathcal{D}$, for all $t \geq 0$. Moreover

$$
\lim _{T \rightarrow \infty}\left\|T K_{T}-L\right\|=0 .
$$

Proof. $L$ is bounded as $\left\|A_{\omega}\right\|$ is square integrable and both $\hat{h}(\omega)$ and $s(\omega)$ are integrable, and it is manifestly completely dissipative. From the stated form of the generator $L$, the fact that $\tau_{t}$ are automorphisms, and that $\tau_{t}\left(A_{\omega}\right)=$ $\mathrm{e}^{-i \omega t} A_{\omega}$, one then sees that

$$
X \in \mathcal{D} \Rightarrow\left(L \tau_{t}\right)(X)=\left(\tau_{t} L\right)(X)=L(X),
$$

proving the invariance of $\mathcal{D}$ under $\mathrm{e}^{L t}$. We pass to the limit $T \rightarrow \infty$ : using $\bar{\omega}=$ $1 / 2 T$ we plug $A_{\omega, T}^{\dagger}=\int \mathrm{d} \omega_{\alpha} \sqrt{\delta_{\bar{\omega}}}\left(\omega-\omega_{\alpha}\right) A_{-\omega_{\alpha}}$ and $A_{\omega, T}=\int \mathrm{d} \omega_{\beta} \sqrt{\delta_{\bar{\omega}}}(\omega-$ $\left.\omega_{\beta}\right) A_{\omega_{\beta}}$ into (18), change variable with $\Omega=\frac{\omega_{\alpha}+\omega_{\beta}}{2}$ and $\nu=\omega_{\alpha}-\omega_{\beta}$, and use (21) to find

$$
\begin{aligned}
\frac{1}{2 \bar{\omega}} K_{\frac{1}{2 \bar{\omega}}}(X)= & -2 \pi i \int \mathrm{d} \Omega s_{\bar{\omega}}(\Omega)\left[\left(A_{-\Omega} A_{\Omega}\right)^{\natural(\bar{\omega})}, X\right] \\
& +2 \pi \int \mathrm{d} \Omega \hat{h}_{\bar{\omega}}(\Omega)\left(-\frac{1}{2}\left\{\left(A_{-\Omega} A_{\Omega}\right)^{\natural(\bar{\omega})}, X\right\}+\left(A_{-\Omega} X A_{\Omega}\right)^{\natural(\bar{\omega})}\right)
\end{aligned}
$$

with

$$
\begin{aligned}
\hat{h}_{\bar{\omega}}(\Omega) & =\int \mathrm{d} \omega \hat{h}(\omega) \delta_{\bar{\omega}}(\Omega-\omega), \\
s_{\bar{\omega}}(\Omega) & =\int \mathrm{d} \omega \hat{h}(\omega) \sqrt{\frac{2}{\pi}} \delta_{\bar{\omega}}(\Omega-\omega) i \operatorname{erf}\left(\frac{i}{\sqrt{2}} \frac{\Omega-\omega}{\bar{\omega}}\right) \\
\left(A_{-\Omega} X A_{\Omega}\right)^{\natural(\bar{\omega})} & =\int \mathrm{d} \nu \delta_{\bar{\omega}}(\nu) A_{-\Omega-\nu} X A_{\Omega-\nu} .
\end{aligned}
$$

Now

$$
\sqrt{\frac{2}{\pi}} \delta_{\bar{\omega}}(\omega) i \operatorname{erf}\left(\frac{i}{\sqrt{2}} \frac{\omega}{\bar{\omega}}\right) \rightarrow \mathcal{P} \frac{1}{\sqrt{2 \pi} \omega}, \quad \bar{\omega} \rightarrow 0^{+}
$$

in the sense of distributions, so that $s_{\bar{\omega}} \rightarrow s$ and $\hat{h}_{\bar{\omega}} \rightarrow \hat{h}$ pointwise as $\bar{\omega} \rightarrow 0^{+}$, because of our hypotheses on $s$ and $\hat{h}$. The fact that $\int \mathrm{d} \omega\left\|A_{\omega}\right\|^{2}<\infty$ furnishes an integrable upper bound for the dominated convergence theorem, so that convergence

$$
\left\|\left(\frac{1}{2 \bar{\omega}} K_{\frac{1}{2 \bar{\omega}}}-\widetilde{L}_{\bar{\omega}}\right)(X)\right\| \rightarrow 0, \quad \bar{\omega} \rightarrow 0^{+},
$$

is proved uniformly on $\|X\|=1$ for the obvious intermediate

$$
\begin{aligned}
\widetilde{L}_{\bar{\omega}}(X)= & -2 \pi i \int \mathrm{d} \omega s(\omega)\left[\left(A_{-\omega} A_{\omega}\right)^{\natural(\bar{\omega})}, X\right] \\
& +2 \pi \int \mathrm{d} \omega \hat{h}(\omega)\left(-\frac{1}{2}\left\{\left(A_{-\omega} A_{\omega}\right)^{\natural(\bar{\omega})}, X\right\}+\left(A_{-\omega} X A_{\omega}\right)^{\natural(\bar{\omega})}\right)
\end{aligned}
$$


(where we renamed $\Omega$ as $\omega$ ). To compare $\widetilde{L}_{\bar{\omega}}(X)$ with $L_{\bar{\omega}}(X)$, we use $\hat{h}$ as an integrable upper bound for the dominated convergence theorem applied to the pointwise convergence

$$
\left\|\left(A_{-\omega} X A_{\omega}\right)^{\natural(\bar{\omega})}-A_{-\omega} X A_{\omega}\right\| \rightarrow 0, \quad \bar{\omega} \rightarrow 0^{+}
$$

In turn, this follows from the fact that the norm of the above difference is smaller than

$$
c\|X\| \int \mathrm{d} \nu \delta_{\bar{\omega}}(\nu)\left(\left\|A_{-\omega-\nu}-A_{-\omega}\right\|+\left\|A_{\omega-\nu}-A_{\omega}\right\|\right),
$$

which goes to zero for $\bar{\omega} \rightarrow 0^{+}$, because $\left\|A_{\omega}-A_{\omega+\nu}\right\| \leq\left\|A_{\omega}\right\|+\left\|A_{\omega+\nu}\right\|$ is uniformly bounded and continuous with respect to $\nu$, due to continuity of $A_{\omega}$ and the asymptotics (23). Proceeding similarly for the commutator part proves that $\left\|\left(\widetilde{L}_{\bar{\omega}}-L\right)(X)\right\| \rightarrow 0$ uniformly on $\|X\|=1$ and thus the first claim of the proposition.

As a remark, we have shown, under the conditions of Proposition 7.5 above and for $\langle\Phi\rangle_{\beta}=0$, that for every $\bar{\tau}>0$

$$
\lim _{\lambda \rightarrow 0}\left\{\sup _{0 \leq t \leq \lambda-2 \bar{\tau}}\left\|P_{0} \mathrm{e}^{(Z+\lambda A) t} P_{0}-\mathrm{e}^{\left(Z_{0}+\frac{\lambda^{3}}{\bar{\tau}} L\right) t}\right\|\right\}=0 .
$$

Indeed assumptions in Theorem 4.4 are satisfied, as proven in [2], and the markovian estimate is deduced from Theorem 4.2 and Proposition 7.5 with $T(\lambda)=|\lambda|^{-1} \bar{\tau}$ for $\lambda \neq 0$.

We are now in position to focus on the case of a free particle in the 3D-euclidean space weakly coupled to a heat bath, for which we shall prove the validity of the general framework above (namely Assumption 7.1 and the hypotheses on $A_{\omega}$ of Proposition 7.5).

As the bath is concerned, we shall assume it to be described, as in [2], by a quasi-free representation on $\mathcal{H}_{B}$ of operators $\varphi(f), f$ in the Hilbert space $\mathcal{V}$ of test functions, satisfying the CAR algebra $\{\varphi(f), \varphi(g)\}=2 \Re\langle f, g\rangle 1$, and being compatible with single particle dynamics in the sense that $U_{t}(1 \otimes \varphi(f))=$ $1 \otimes \varphi\left(\mathrm{e}^{i S t} f\right)$ for a (densely defined) self-adjoint operator $S$ on $\mathcal{V}$. Moreover $|\Omega\rangle$ is cyclic, $H_{B}|\Omega\rangle=0$, and the representation is fixed by

$$
\langle\Omega, \varphi(f) \varphi(g) \Omega\rangle=\left\langle g, \mathrm{e}^{-\beta S}\left(1+\mathrm{e}^{-\beta S}\right)^{-1} f\right\rangle+\left\langle f,\left(1-\mathrm{e}^{-\beta S}\left(1+\mathrm{e}^{-\beta S}\right)^{-1}\right) g\right\rangle
$$

for inverse temperature $\beta$. The perturbation $H^{\prime}=Q \otimes \Phi$ is chosen with

$$
\Phi=i \varphi\left(f_{1}\right) \varphi\left(f_{-1}\right),
$$

for some $f_{1}$ and $f_{-1}$ in $\mathcal{V}$. Even though for what follows it will be sufficient to assume that $f_{ \pm 1}$ have disjoint energy spectra, implying $\langle\Phi\rangle_{\sigma}=0$ (see [2]), possible first-order contributions could be included, as we report here in a slight generalization of Lemma 4.1 in [2].

Lemma 7.6. If $h$ is integrable then its transform satisfies

$$
\hat{h}(-\omega)=\mathrm{e}^{-\beta \omega} \hat{h}(\omega)
$$

for all $\omega \in \mathbb{R}$. 
Proof. By quasi-freeness, Wick expansion of $h(t)$ gives

$$
h(t)=h_{1}(t) h_{-1}(t)-|\chi(t)|^{2}
$$

with $h_{i}(t)=\left\langle\Omega, \varphi\left(\mathrm{e}^{i S t} f_{i}\right) \varphi\left(f_{i}\right) \Omega\right\rangle$ and $\chi(t)=\left\langle\Omega, \varphi\left(\mathrm{e}^{i S t} f_{1}\right) \varphi\left(f_{-1}\right) \Omega\right\rangle$. The spectral theorem and (24) give $\hat{h}_{i}(-\omega)=\mathrm{e}^{-\beta \omega} \hat{h}_{i}(\omega)$ and $\widetilde{\chi}(-\omega)=\mathrm{e}^{-\beta \omega} \widetilde{\chi}(\omega)^{*}$, so that the conclusion follows from

$$
\hat{h}(\omega)=\int \mathrm{d} \omega^{\prime}\left(\hat{h}_{1}\left(\omega^{\prime}\right) \hat{h}_{-1}\left(\omega-\omega^{\prime}\right)-\widetilde{\chi}\left(\omega^{\prime}\right)^{*} \widetilde{\chi}\left(\omega-\omega^{\prime}\right)\right) .
$$

In [2], the conditions $\int_{0}^{\infty}\left|h_{i}(t)\right|\left(1+t^{\epsilon}\right) \mathrm{d} t<\infty$ are shown to be sufficient for the hypotheses of Theorem 4.4 (and Lemma 7.6) to hold, in case $\chi(t)$ vanishes identically. We conjecture that there exist analogous conditions on $\chi(t)$ for the foretold hypotheses to hold, but leave the involved analysis to future work and, as already stated, we shall assume $\chi \equiv 0$.

We now focus on the particle, by specializing the coupling to depend on energy transitions only:

Proposition 7.7. Let $\mathcal{H}=\mathcal{L}^{2}\left(\mathbb{R}^{3}\right)$ be the Hilbert space of a free particle $H_{A}=P^{2} / 2$ in three dimensions, and let the integral kernel $q\left(p, p^{\prime}\right)$ define (the bounded self-adjoint operator) $Q$ in the representation where $P$ is the multiplication operator. Let $q\left(p, p^{\prime}\right)=\widetilde{q}\left(\varepsilon_{p}, \varepsilon_{p^{\prime}}\right)$ for a complex function $\widetilde{q}$ in the Schwartz space $\mathcal{S}\left(\mathbb{R}^{2}\right)$, and $\varepsilon_{p}=p^{2} / 2$. Then Assumptions 7.1 and the hypotheses on $A_{\omega}$ of Proposition 7.5 are satisfied.

Proof. Using the spectral theorem for $\tau_{t}(Q)$, Assumption 7.1 is satisfied if for test functions $\phi, \psi$ in the Schwartz space $\mathcal{S}$ (and denoting with $\theta$ the Heaviside step function) the equality

$$
\left\langle\phi, A_{\omega} \psi\right\rangle=\iint \mathrm{d}^{3} p \mathrm{~d}^{3} p^{\prime} \delta\left(\omega-\left(\varepsilon_{p}-\varepsilon_{p^{\prime}}\right)\right) q\left(p, p^{\prime}\right) \phi(p)^{*} \psi\left(p^{\prime}\right),
$$

(i) defines an operator $A_{\omega}$ on $\mathcal{S}\left(\mathbb{R}^{3}\right)$; (ii) $A_{\omega}$ extends to a bounded operator on $\mathcal{H}$ (that we again denote with $A_{\omega}$ ); (iii) the integral at the right hand side of (17) is bounded.

Now, point (i) follows, as the surfaces $S_{\varepsilon}=\left\{p^{\prime} \in \mathcal{R}^{3} \mid \varepsilon=\omega+\varepsilon_{p^{\prime}}\right\}$ are regular and compact (they are possibly degenerate spheres), the function $\widetilde{q}$ is in Schwartz space, the gradient $\nabla \varepsilon_{p}$ is zero only at the origin, and for every real $\omega$ the singularity is controlled by the jacobian passing to spherical coordinates.

Point (ii) follows if $\left\|A_{\omega} \psi\right\|$ is bounded uniformly on normalized $\psi \in$ $\mathcal{S}\left(\mathbb{R}^{3}\right)$, because of density. We compute 


$$
\begin{aligned}
\left\|A_{\omega} \psi\right\|^{2}= & \iint \mathrm{d}^{3} p \mathrm{~d}^{3} p^{\prime} \delta\left(\varepsilon_{p}-\varepsilon_{p^{\prime}}\right)\left|\widetilde{q}\left(\varepsilon_{p}, \varepsilon_{p}+\omega\right)\right|^{2} \eta\left(\omega+\varepsilon_{p}\right) \psi\left(p^{\prime}\right)^{*} \psi(p) \\
= & \int_{0}^{\infty} \mathrm{d} \xi \xi^{3} \eta\left(\omega+\xi^{2} / 2\right)\left|\widetilde{q}\left(\xi^{2} / 2, \omega+\xi^{2} / 2\right)\right|^{2} \\
& \times\left|\iint \sin \theta \mathrm{d} \theta \mathrm{d} \phi \psi(\xi, \theta, \phi)\right|^{2}
\end{aligned}
$$

with $\eta(\varepsilon)=4 \pi \theta(\varepsilon) \sqrt{2|\varepsilon|}$ being the density of states, and having passed to spherical coordinates in the second and third lines. The third line is overestimated by $4 \pi \iint \sin ^{2} \theta \mathrm{d} \theta \mathrm{d} \phi|\psi(\xi, \theta, \phi)|^{2}$, so that

$$
\left\|A_{\omega} \psi\right\|^{2} \leq \int \mathrm{d}^{3} p f\left(\varepsilon_{p}, \omega+\varepsilon_{p}\right)|\psi(p)|^{2}
$$

with continuous $f\left(\varepsilon, \varepsilon^{\prime}\right)=\eta(\varepsilon) \eta\left(\varepsilon^{\prime}\right)\left|\widetilde{q}\left(\varepsilon, \varepsilon^{\prime}\right)\right|^{2}$. Now, for any $\omega$ the right hand side above is smaller than $c\|\psi\|^{2}$ for some constant $c$ independent of $\psi$, because $\widetilde{q}$ is continuous and $\widetilde{q}\left(\varepsilon, \varepsilon^{\prime}\right)=o(\varepsilon)$ for $\varepsilon \rightarrow+\infty$, and $\eta(\varepsilon)=O(1)$ for $\varepsilon \rightarrow 0+$.

Point (iii) follows from the fact that the function $\omega \mapsto A_{\omega}$ is (a) continuous, and (b) $\left\|A_{\omega}\right\|=o(\omega+\epsilon)$ for some $\epsilon>0$ and for $|\omega| \rightarrow \infty$. To prove point (a), denote with $\partial_{1} \widetilde{q}\left(\varepsilon, \varepsilon^{\prime}\right)$ the partial derivative with respect to the first argument, and compute

$$
\begin{aligned}
\left\|\left(A_{\omega+\nu}-A_{\omega}\right) \psi\right\|^{2}= & \nu^{2} \iint \mathrm{d}^{3} p \mathrm{~d}^{3} p^{\prime} \delta\left(\varepsilon_{p}-\varepsilon_{p^{\prime}}\right)\left|\partial_{1} \widetilde{q}\left(\varepsilon_{p}, \varepsilon_{p}+\omega\right)\right|^{2} \eta\left(\omega+\varepsilon_{p}\right) \\
& \times \psi\left(p^{\prime}\right)^{*} \psi(p)+o\left(\nu^{2}\right)
\end{aligned}
$$

due to continuity of $\eta$, and the fact that $p \mapsto \widetilde{q}\left(\varepsilon_{p}, \varepsilon_{p}+\omega\right)$ is in $\mathcal{S}\left(\mathbb{R}^{3}\right)$, and proceeding as above shows that the second-order term in $\nu$ is bounded at every real $\omega$ because $\widetilde{q} \in \mathcal{S}\left(\mathbb{R}^{3}\right)$.

Now, $b$ ) follows because $f(\varepsilon, \varepsilon+\omega)=o\left(\omega^{2}+\epsilon^{+}\right)$as $|\omega| \rightarrow \infty$ uniformly on all $\varepsilon \in \mathbb{R}^{+}$, as can be seen directly for $\varepsilon \rightarrow 0^{+}$, and by noting that $\bar{q}(\varepsilon, \omega):=$ $\widetilde{q}(\varepsilon, \varepsilon+\omega)$ is also in Schwartz space. Finally, (26) shows that $\tau_{t}\left(A_{\omega}\right)=\mathrm{e}^{-i \omega t} A_{\omega}$ for all $t, \omega \in \mathbb{R}$. This proves the validity of Assumption 7.1 and at the same time all the other requirements on $A_{\omega}$, concluding the proof.

We now consider the problem of stationary distribution for (at least a sufficiently ample subclass of ) elements of $\mathcal{D}=\left\{X \in \mathcal{B}\left(\mathcal{H}_{A}\right) \mid \tau_{t}(X)=X, \forall t \in \mathbb{R}\right\}$.

In $\mathcal{T}\left(\mathcal{H}_{A}\right)$ consider the equivalence relation $\rho_{1} \sim \rho_{2}$ iff $\operatorname{Tr}\left(\rho_{1} A\right)=\operatorname{Tr}\left(\rho_{2} A\right)$ for all $A \in \mathcal{D}$, and for $\rho \in \mathcal{T}\left(\mathcal{H}_{A}\right)$ denote with $[\rho]$ its associated equivalence class. Then clearly $\operatorname{Tr}(\rho A)=\operatorname{Tr}([\rho] A)$ passes to the quotient for $A \in \mathcal{D}$.

Let $\rho_{\beta}$ be the density matrix (positive trace-class normalized operator) on $\mathcal{H}_{A}$, whose kernel in the momentum representation is given by

$$
\rho_{\beta}\left(p_{1}, p_{2}\right)=\sqrt{\frac{\mu_{\beta}\left(\varepsilon_{p_{1}}\right)}{\eta\left(\varepsilon_{p_{1}}\right)}} \sqrt{\frac{\mu_{\beta}\left(\varepsilon_{p_{2}}\right)}{\eta\left(\varepsilon_{p_{2}}\right)}} \text { for } \mu_{\beta}(\varepsilon)=\frac{\mathrm{e}^{-\beta \varepsilon}}{\int_{0}^{\infty} \mathrm{e}^{-\beta \varepsilon^{\prime}} \mathrm{d} \varepsilon^{\prime}}
$$

and $\eta(\varepsilon)=4 \pi \sqrt{2 \varepsilon}$ being the density of states. 
Define the subset $\mathcal{G} \subset \mathcal{D}$ of bounded operators $G$ on $\mathcal{H}_{A}$ whose kernel in momentum representation has the form $G\left(p_{1}, p_{2}\right)=g\left(p_{1}, p_{2}\right) \delta\left(\varepsilon_{p_{1}}-\varepsilon_{p_{2}}\right)$ for some $g \in \mathcal{S}\left(\mathbb{R}^{6}\right)$, and for $G \in \mathcal{G}$ define the $L^{\infty}\left(\mathbb{R}^{+}\right)$function $\widetilde{g}$ by

$$
\widetilde{g}(\varepsilon):=\iint \mathrm{d} p_{1} \mathrm{~d} p_{2} g\left(p_{1}, p_{2}\right) \delta\left(\varepsilon-\varepsilon_{p_{1}}\right) \delta\left(\varepsilon-\varepsilon_{p_{2}}\right) .
$$

Proposition 7.8. Under the setting of Proposition 7.7, let moreover $\hat{h}$ be in the Schwartz space $\mathcal{S}(\mathbb{R})$. Then for all $G \in \mathcal{G}$, under Definitions 27 and 28 , the expectation

$$
\langle G\rangle_{\beta}(t):=\operatorname{Tr}\left(\left[\rho_{\beta}\right] \mathrm{e}^{\left(Z_{0}+\frac{\lambda^{3}}{\bar{\tau}} L\right) t} G\right)=\int_{0}^{\infty} \widetilde{g}(\varepsilon) \mu_{\beta}(\varepsilon) \mathrm{d} \varepsilon
$$

is stationary (for all $\bar{\tau}>0$ ).

Proof. Clearly $\left.Z_{0}\right|_{\mathcal{D}}=0$, so $\langle G\rangle_{\beta}(t)=\operatorname{Tr}\left(\left[\rho_{\beta}\right] \mathrm{e}^{L t} G\right)$ modulo rescaling time. Using bracket notation, the spectral decomposition

$$
A_{\omega}=\iint \delta\left(\omega+\varepsilon-\varepsilon^{\prime}\right) \mathrm{d} E_{\varepsilon} Q \mathrm{~d} E_{\varepsilon^{\prime}}
$$

for $H_{A}=\int \varepsilon \mathrm{d} E_{\varepsilon}$ allows to compute $L G$ according to

$$
\begin{aligned}
\int \mathrm{d} \omega \hat{h}(\omega) A_{\omega}^{\dagger} A_{\omega} G= & \int \mathrm{d} \omega \hat{h}(\omega) G A_{\omega}^{\dagger} A_{\omega} \\
= & \iint \mathrm{d} p \mathrm{~d} p_{1}|p\rangle\left\langle\left. p_{1}\left|\delta\left(\varepsilon_{p}-\varepsilon_{p_{1}}\right)\right| \widetilde{q}\left(\varepsilon_{p}, \varepsilon_{p^{\prime}}\right)\right|^{2} \hat{h}\left(\varepsilon_{p}-\varepsilon_{p^{\prime}}\right)\right. \\
& \times \int \mathrm{d} p_{2} g\left(p_{2}, p_{1}\right) \delta\left(\varepsilon_{p_{1}}-\varepsilon_{p_{2}}\right)
\end{aligned}
$$

and

$$
\begin{aligned}
\int \mathrm{d} \omega \hat{h}(\omega) A_{\omega}^{\dagger} G A_{\omega}= & \iint \mathrm{d} p \mathrm{~d} p_{1}|p\rangle\left\langle\left. p_{1}\left|\delta\left(\varepsilon_{p}-\varepsilon_{p_{1}}\right)\right| \widetilde{q}\left(\varepsilon_{p}, \varepsilon_{p^{\prime}}\right)\right|^{2} \hat{h}\left(\varepsilon_{p}-\varepsilon_{p^{\prime}}\right)\right. \\
& \times \int \mathrm{d} p_{2} g\left(p_{2}, p^{\prime}\right) \delta\left(\varepsilon_{p^{\prime}}-\varepsilon_{p_{2}}\right) .
\end{aligned}
$$

From these expressions, and from the regularity assumptions for $\widetilde{q}$ and $\hat{h}$, we see that $\mathrm{e}^{L t}$ preserves $\mathcal{G}$ at all (positive) times. Moreover, we compute

$$
\operatorname{Tr}\left(\left[\rho_{\beta}\right] L G\right)=2 \pi \iint \mathrm{d} \varepsilon \mathrm{d} \varepsilon^{\prime} \mu_{\beta}(\varepsilon) \hat{h}\left(\varepsilon-\varepsilon^{\prime}\right)\left|q\left(\varepsilon, \varepsilon^{\prime}\right)\right|^{2}\left(\eta(\varepsilon) \widetilde{g}\left(\varepsilon^{\prime}\right)-\eta\left(\varepsilon^{\prime}\right) \widetilde{g}(\varepsilon)\right),
$$

or equivalently $\operatorname{Tr}\left(\left[\rho_{\beta}\right] L G\right)=\int \mathrm{d} \varepsilon \widetilde{g}(\varepsilon)\left(L^{*} \mu_{\beta}\right)(\varepsilon)$ where $L^{*}$ acting on $L^{1}\left(\mathbb{R}^{+}\right)$ gives the Boltzmann equation

$$
\left(L^{*} \mu\right)(\varepsilon)=\int_{0}^{\infty} \mathrm{d} \varepsilon^{\prime} \eta\left(\varepsilon^{\prime}\right)\left(r\left(\varepsilon^{\prime}, \varepsilon\right) \mu\left(\varepsilon^{\prime}\right)-r\left(\varepsilon, \varepsilon^{\prime}\right) \mu(\varepsilon)\right)
$$

with celebrated Fermi Golden Rule transition rates

$$
r\left(\varepsilon^{\prime}, \varepsilon\right)=2 \pi \hat{h}\left(\varepsilon^{\prime}-\varepsilon\right)\left|\widetilde{q}\left(\varepsilon, \varepsilon^{\prime}\right)\right|^{2} .
$$


The statement of the proposition then follows from the detailed balance condition

$$
r\left(\varepsilon, \varepsilon^{\prime}\right)=\mathrm{e}^{\beta\left(\varepsilon-\varepsilon^{\prime}\right)} r\left(\varepsilon^{\prime}, \varepsilon\right)
$$

due to Lemma 7.6 (and then by explicit computation of $\operatorname{Tr}\left(\left[\rho_{\beta}\right] G\right)$ ).

Remarks. - The above proposition only needs the function $\widetilde{g}$ to be in $L^{\infty}\left(\mathbb{R}^{+}\right)$, surely allowing to include a class of observables in $\mathcal{D}$ larger than $\mathcal{G}$ : for example, bounded and sufficiently regular functions of the energy should be included without problems, even if they don't belong to $\mathcal{G}$.

- Unicity of the steady distribution for $G \in \mathcal{G}$ is not obvious from the above analysis, as the fact that $\operatorname{Tr}\left(\left[\rho_{\beta}\right] L G\right)=\int \mathrm{d} \varepsilon \widetilde{g}(\varepsilon)\left(L^{*} \mu_{\beta}\right)(\varepsilon)$ depends on $G$ only through $\widetilde{g}(\varepsilon)$ is conditional on $\left[\rho_{\beta}\right]$, and it is not generally true for other density matrices. We argue that unicity could be restored for momentum conserving interactions $H^{\prime}$, but this would take us beyond the scope of the present example (whose aim is to motivate the general theory section through a simple analysis).

- There are no KMS states for a free particle in Euclidean space (physically, one cannot measure position if the particle is "everywhere"), and therefore one is forced to consider equilibrium distribution for a suitable subclass of observables only. ${ }^{4}$

\section{Proofs of Theorems 3.4 and 4.2}

The following lemma is not new, and for example it is contained in Theorem 1.2 of [3], but we report it here as we shall make use of it repeatedly all throughout.

Lemma 8.1. Let $b \in \mathcal{B}_{0}$ be given, together with some real $\bar{\tau}>0$. Suppose $X_{t}^{\lambda}$ is a one-parameter group of isometries. Suppose $W_{t}^{\lambda}$ and $\bar{W}_{t}^{\lambda}$ are operators on $\mathcal{B}_{0}$ such that $f_{\lambda}(\tau)=X_{-\lambda^{-2} \tau^{\lambda}} W_{\lambda^{-2} \tau}^{\lambda}$ b satisfies

$$
f_{\lambda}=\sum_{n \geq 0} \mathcal{H}_{\lambda}^{n} b
$$

for a Volterra operator $\mathcal{H}_{\lambda}$ on the Banach space $\mathcal{V}=\mathcal{C}^{0}\left([0, \bar{\tau}], \mathcal{B}_{0}\right)$ of continuous $\mathcal{B}_{0}$-valued functions on the interval $[0, \bar{\tau}]$ (assume the same holds also for $\bar{W}_{t}^{\lambda}$, with associated $\bar{f}_{\lambda}$ and $\left.\overline{\mathcal{H}}_{\lambda}\right)$. Suppose there exists a real positive $c$ such that $\left\|\mathcal{H}_{\lambda}\right\|_{\mathcal{V}} \leq c \bar{\tau}$ and $\left\|\overline{\mathcal{H}}_{\lambda}\right\|_{\mathcal{V}} \leq c \bar{\tau}$ uniformly on $|\lambda| \leq 1$. Put

(i) $\lim _{\lambda \rightarrow 0}\left\|\mathcal{H}_{\lambda}-\overline{\mathcal{H}}_{\lambda}\right\|_{\mathcal{V}}=0$;

(ii) $\lim _{\lambda \rightarrow 0}\left\|f_{\lambda}-\bar{f}_{\lambda}\right\|_{\infty}=0$;

(iii) $\lim _{\lambda \rightarrow 0} \sup _{0 \leq t \leq \lambda-2 \bar{\tau}}\left\|W_{t}^{\lambda} b-\bar{W}_{t}^{\lambda} b\right\|_{\mathcal{B}_{0}}=0$.

Then (i) $\Rightarrow$ (ii) $\Rightarrow$ (iii).

\footnotetext{
${ }^{4}$ We are grateful to our referee for pointing that out.
} 
Proof. Of course (ii) $\Rightarrow$ (iii) as $X_{t}^{\lambda}$ is group of isometries and one has

$$
\sup _{0 \leq t \leq \lambda^{-2} \bar{\tau}}\left\|W_{t}^{\lambda} b-\bar{W}_{t}^{\lambda} b\right\|_{\mathcal{B}_{0}}=\sup _{0 \leq \tau \leq \bar{\tau}}\left\|f_{\lambda}(\tau)-\bar{f}_{\lambda}(\tau)\right\|_{\mathcal{B}_{0}}=\left\|f_{\lambda}-\bar{f}_{\lambda}\right\|_{\infty} .
$$

Then, subtracting the von Newmann expansions for $f_{\lambda}$ and $\bar{f}_{\lambda}$ one obtains

$$
\begin{aligned}
\left\|f_{\lambda}-\bar{f}_{\lambda}\right\|_{\infty} & \leq \sum_{n=1}^{\infty}\left\|\mathcal{H}_{\lambda}^{n} b-\overline{\mathcal{H}}_{\lambda}^{n} b\right\|_{\infty} \\
& =\sum_{n=1}^{\infty}\left\|\mathcal{H}_{\lambda}^{n-1}\left(\mathcal{H}_{\lambda}-\overline{\mathcal{H}}_{\lambda}\right) b+\cdots+\left(\mathcal{H}_{\lambda}-\overline{\mathcal{H}}_{\lambda}\right) \overline{\mathcal{H}}_{\lambda}^{n-1} b\right\|_{\infty} \\
& \leq\left\|\mathcal{H}_{\lambda}-\overline{\mathcal{H}}_{\lambda}\right\|_{\mathcal{V}}\|b\|_{\mathcal{B}_{0}} \sum_{n=1}^{\infty} \frac{(\bar{\tau} c)^{n-1}}{(n-1) !}
\end{aligned}
$$

and the last series is (obviously) convergent and independent of $\lambda$. Note that we have used (and will use throughout) the important property that if $\mathcal{H}$ is Volterra and $\|\mathcal{H}\| \leq C$, then $\left\|\mathcal{H}^{n}\right\| \leq C^{n} / n$ ! (see e.g. Eq. (1.28) in [3]).

This shows that (i) $\Rightarrow$ (ii) and thus finishes the proof.

We still need a technical result that will allow us to perform approximation (8): its interpretation will become clear in the context of the proof of Theorem 3.4, but it deserves to be reported in the more autonomous environment of a Lemma, as it will find application also in the proof of Theorem 4.2.

Lemma 8.2. Let $\mathcal{B}_{0}$ be a Banach space, $\bar{\tau}>0$, and let $\mathcal{V}=\mathcal{C}^{0}\left([0, \bar{\tau}], \mathcal{B}_{0}\right)$ be the Banach space of continuous functions from $[0, \bar{\tau}]$ into $\mathcal{B}_{0}$. For some real $q$ and $\alpha$, let $\mathcal{D}(\lambda, \tau, \alpha, q)$ be the triangle in the $(\sigma, x)$-plane of vertices

$$
\mathcal{D}(\lambda, \tau, \alpha, q)=\triangle\left\{\left(\lambda^{2} q, 0\right),\left(\tau+\lambda^{2} q, 0\right),\left(\left(\frac{1}{2}-\alpha\right) \tau+\lambda^{2} q, \lambda^{-2} \tau\right)\right\}
$$

and define the truncated domain ${ }^{5}$ (see Fig. 1)

$$
\overline{\mathcal{D}}(\lambda, \tau, \alpha, q)=\mathcal{D}(\lambda, \tau, \alpha, q) \cap[0, \tau] \times[0, \infty) .
$$

Let $T: \dot{I} \rightarrow \mathbb{R}^{+}$a real positive continuous function, assume

$$
T(\lambda) \sim|\lambda|^{-\xi} \widetilde{T}, \quad \lambda \rightarrow 0
$$

for some $\widetilde{T}>0$ and $0<\xi<2$ (strictly). Let $\mathcal{H}_{(\lambda \alpha q)}$ be a Volterra integral operator on $\mathcal{V}$ and assume it can be put in the form

$$
\left(\mathcal{H}_{(\lambda \alpha q)} g\right)(\tau)=\iint_{\overline{\mathcal{D}}(\lambda, \tau, \alpha, q)} \mathrm{d} \sigma \mathrm{d} x \mathrm{e}^{-\frac{(x / 2)^{2}}{2 T(\lambda)^{2}}} K_{\alpha q}(\lambda, \sigma, x) g\left(\sigma-\lambda^{2}(q+(1 / 2-\alpha) x)\right),
$$

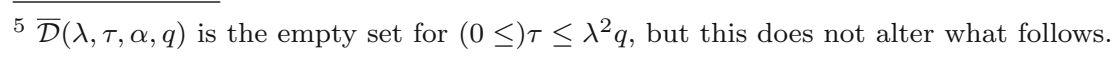


for a suitable kernel $K_{\alpha q}(\lambda, \sigma, x)$, and define the Volterra integral operator $\overline{\mathcal{H}}_{(\lambda \alpha q)}$ by

$$
\left(\overline{\mathcal{H}}_{(\lambda \alpha q)} g\right)(\tau)=\iint_{\overline{\mathcal{D}}(\lambda, \tau, \alpha, q)} \mathrm{d} \sigma \mathrm{d} x \mathrm{e}^{-\frac{(x / 2)^{2}}{2 T(\lambda)^{2}}} K_{\alpha q}(\lambda, \sigma, x) g(\sigma) .
$$

Suppose that $\left\|K_{\alpha q}(\lambda, \sigma, x)\right\|=k(x)$ independently on $\sigma, \lambda, \alpha, q$, and that

$$
\int_{0}^{\infty} \mathrm{d} x k(x)=c
$$

for some finite $0<c<\infty$, and assume $\left\|\mathcal{H}_{(\lambda \alpha q)}\right\|<\bar{\tau} c$ uniformly on $|\lambda| \leq 1$.

Let $b \in \mathcal{B}_{0}$ and define the von Neumann series $f_{(\lambda \alpha q)}$ and $\bar{f}_{(\lambda \alpha q)}$ through (32) in Lemma 8.1.

Then

$$
\left\|\overline{\mathcal{H}}_{(\lambda \alpha q)}\right\| \leq \bar{\tau} c
$$

uniformly on $|\lambda| \leq 1$ and

$$
\lim _{\lambda \rightarrow 0}\left\|f_{(\lambda \alpha q)}-\bar{f}_{(\lambda \alpha q)}\right\|_{\infty}=0 .
$$

Proof. Estimation (36) is a consequence of the definition of $\overline{\mathcal{H}}_{(\lambda \alpha q)}$, as

$$
\left\|\overline{\mathcal{H}}_{(\lambda \alpha q)} g(\tau)\right\| \leq \tau \int_{0}^{\infty} k(x)\|g\|_{\infty} .
$$

To show the validity of (37) we perform a telescopic expansion as in the proof of Lemma 8.1 to obtain the following estimate,

$$
\left\|f_{(\lambda \alpha q)}-\bar{f}_{(\lambda \alpha q)}\right\|_{\infty} \leq \sum_{n=1}^{\infty} \sum_{l=1}^{n-1} \frac{(\bar{\tau} c)^{n-l-1}}{(n-l) !}\left\|\left(\mathcal{H}_{(\lambda \alpha q)}-\overline{\mathcal{H}}_{(\lambda \alpha q)}\right) \overline{\mathcal{H}}_{(\lambda \alpha q)}^{l} b\right\|_{\infty} .
$$

Note that the case $l=0$ has been dropped, since $\mathcal{H}_{(\lambda \alpha q)} b=\overline{\mathcal{H}}_{(\lambda \alpha q)} b$ trivially, as $b$ is constant, as one can see from (34). Now if we could show that for every $\epsilon>0$ there exists some $\bar{\lambda}>0$ such that $|\lambda|<\bar{\lambda}$ implies that for every $l \geq 1$

$$
\left\|\left(\mathcal{H}_{(\lambda \alpha q)}-\overline{\mathcal{H}}_{(\lambda \alpha q)}\right) \overline{\mathcal{H}}_{(\lambda \alpha q)}^{l} b\right\|_{\infty} \leq \frac{(\bar{\tau} c)^{l-1}}{(l-1) !} \bar{\tau}\|b\|_{\mathcal{B}_{0}} \epsilon
$$

we would be done, as, following estimation (38), we would have

$$
\left\|f_{\lambda}-\bar{f}_{\lambda}\right\|_{\infty} \leq \sum_{n=1}^{\infty} \sum_{l=1}^{n-1} \frac{(\bar{\tau} c)^{n-2}}{(n-l) !(l-1) !} \bar{\tau}\|b\|_{\mathcal{B}_{0}} \epsilon
$$




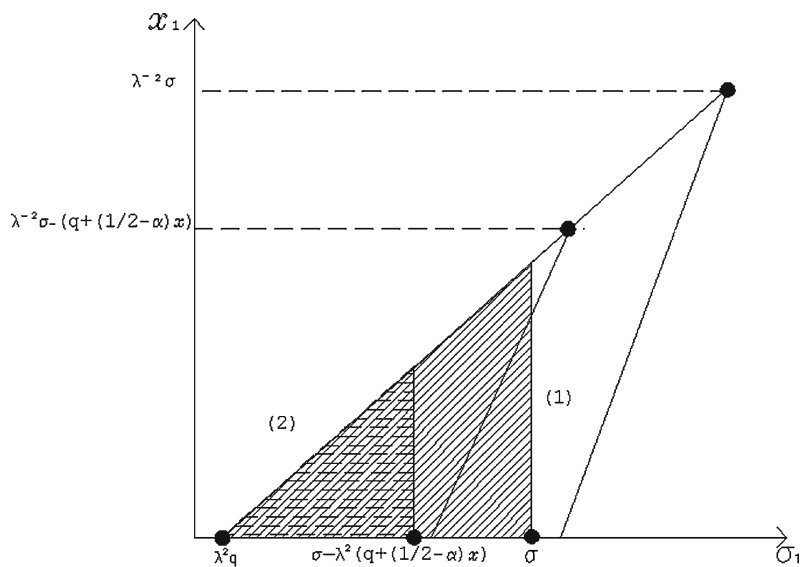

FiguRE 2. Integration domains $\overline{\mathcal{D}}(\lambda, \sigma, \alpha, q)$ (rectangular triangle $(1)), \overline{\mathcal{D}}\left(\lambda, \sigma-\lambda^{2}(q+(1 / 2-\alpha) x), \alpha, q\right)$ (rectangular triangle (2)) and $S(\lambda, \sigma, x)$ (region $(1) \cup(2)=$ region (1) in this case). We have put $\alpha<-1 / 2, q>0$ for clarity. The graph shows that the projection on the $\sigma_{1}$-axis of integration domain $(1) \backslash(2) \rightarrow 0$ as $\lambda \rightarrow 0$. Its complementary projection tends to the whole positive part of the $x_{1}$-axis, but it is controlled by the boundedness of the corresponding integral kernels

and the series would obviously converge (because $c>0$ by hypothesis). To show that property (38) holds, we take $l>0$ and evaluate ${ }^{6}$

$$
\begin{aligned}
& {\left[\left(\mathcal{H}_{(\lambda \alpha q)}-\overline{\mathcal{H}}_{(\lambda \alpha q)}\right) \overline{\mathcal{H}}_{(\lambda \alpha q)}^{l} b\right](\tau)} \\
& =\iint \mathrm{d} \sigma \mathrm{d} x \mathrm{e}^{-\frac{(x / 2)^{2}}{2 T(\lambda)^{2}}} K_{\alpha q}(\lambda, \sigma, x) \\
& \times\left\{\left(\overline{\mathcal{H}}_{(\lambda \alpha q)}^{l} b\right)\left(\sigma-\lambda^{2}(q+(1 / 2-\alpha) x)\right)-\left(\overline{\mathcal{H}}_{(\lambda \alpha q)}^{l} b\right)(\sigma)\right\} \\
& =\iint_{\overline{\mathcal{D}}(\lambda, \tau, \alpha, q)} \mathrm{d} \sigma \mathrm{d} x \mathrm{e}^{-\frac{(x / 2)^{2}}{2 T(\lambda)^{2}}} K_{\alpha q}(\lambda, \sigma, x) \\
& \times \iint_{S(\lambda, \alpha, q, \sigma, x)} \mathrm{d} \sigma_{1} \mathrm{~d} x_{1} \Delta^{\lambda}\left(\sigma_{1}, x_{1}\right) \mathrm{e}^{-\frac{(x / 2)^{2}}{2 T(\lambda)^{2}}} K_{\alpha q}\left(\lambda, \sigma_{1}, x_{1}\right)\left(\overline{\mathcal{H}}_{(\lambda \alpha q)}^{l-1} b\right)\left(\sigma_{1}\right)
\end{aligned}
$$

where we have put $\Delta^{\lambda}=\chi_{1}^{\lambda}-\chi_{2}^{\lambda}, \chi_{1}^{\lambda}$ being the characteristic function of $\overline{\mathcal{D}}\left(\lambda, \sigma-\lambda^{2}\left(q+\left(\frac{1}{2}-\alpha\right) x\right), \alpha, q\right), \chi_{2}^{\lambda}$ the characteristic function of $\overline{\mathcal{D}}(\lambda, \sigma, \alpha, q)$, and we have defined (see Fig. 2)

$$
S(\lambda, \alpha, q, \sigma, x)=\overline{\mathcal{D}}\left(\lambda, \sigma-\lambda^{2}(q+(1 / 2-\alpha) x), \alpha, q\right) \cup \overline{\mathcal{D}}(\lambda, \sigma, \alpha, q) .
$$

${ }^{6}$ Note that $\sigma-\lambda^{2}\left(q+\left(\frac{1}{2}-\alpha\right) x\right)=u \geq 0$. 
Passing to the norms, we overestimate the norm of the left hand side of (38) with

$$
\begin{aligned}
& \sup _{0 \leq \tau \leq \bar{\tau}} \iint_{\overline{\mathcal{D}}(\lambda, \tau, \alpha, q)} \mathrm{d} \sigma \mathrm{d} x \mathrm{e}^{-\frac{(x / 2)^{2}}{2 T(\lambda)^{2}}} k(x) \iint_{S(\lambda, \alpha, q, \sigma, x)} \mathrm{d} \sigma_{1} \mathrm{~d} x_{1}\left|\Delta^{\lambda}\left(\sigma_{1}, x_{1}\right)\right| k\left(x_{1}\right)\left\|\overline{\mathcal{H}}_{(\lambda \alpha q)}^{l-1} b\right\|_{\infty} \\
& \leq \frac{(\bar{\tau} c)^{l-1}}{(l-1) !}\|b\|_{\mathcal{B}_{0}} \sup _{0 \leq \tau \leq \bar{\tau}} \iint_{\overline{\mathcal{D}}(\lambda, \tau, \alpha, q)} \mathrm{d} \sigma \mathrm{d} x \mathrm{e}^{-\frac{(x / 2)^{2}}{2 T(\lambda)^{2}}} k(x) \Xi_{\alpha q}(\lambda, \sigma, x) \\
& \quad \leq \frac{(\bar{\tau} c)^{l-1}}{(l-1) !}\|b\|_{\mathcal{B}_{0}} \bar{\tau} \sup _{0 \leq \sigma \leq \bar{\tau}} \int_{0}^{\infty} \mathrm{d} x \mathrm{e}^{-\frac{(x / 2)^{2}}{2 T(\lambda)^{2}}} k(x) \Xi_{\alpha q}(\lambda, \sigma, x)
\end{aligned}
$$

where we named

$$
\Xi_{\alpha q}(\lambda, \sigma, x)=\iint_{S(\lambda, \alpha, q, \sigma, x)} \mathrm{d} \sigma_{1} \mathrm{~d} x_{1}\left|\Delta^{\lambda}\left(\sigma_{1}, x_{1}\right)\right| k\left(x_{1}\right)
$$

At this point let us consider the sector defined by $q>0$ and $\alpha<-1 / 2$. In this case, it is but a straightforward algebra to show that the two triangles $\overline{\mathcal{D}}\left(\lambda, \sigma-\lambda^{2}(q+(1 / 2-\alpha) x), \alpha, q\right)$ and $\overline{\mathcal{D}}(\lambda, \sigma, \alpha, q)$ are similar (the same is true for the unbarred $\mathcal{D}$ s for arbitrary $(\alpha, q))$ and that their left edges lie on the same line of equation

$$
\bar{x}_{l}\left(\lambda, \sigma_{1}\right)=\frac{\lambda^{-2}}{\frac{1}{2}-\alpha}\left(\sigma_{1}-\lambda^{2} q\right)
$$

in the $\left(\sigma_{1}, x_{1}\right)$-plane (see Fig. 2). In particular $\overline{\mathcal{D}}(\lambda, \sigma, \alpha, q)$ contains $\overline{\mathcal{D}}\left(\lambda, \sigma-\lambda^{2}(q+(1 / 2-\alpha) x), \alpha, q\right)$. For this reason we compute

$$
\begin{aligned}
\Xi_{\alpha q}(\lambda, \sigma, x) & =\int_{\sigma-\lambda^{2}\left(q+\left(\frac{1}{2}-\alpha\right) x\right)}^{\sigma} \mathrm{d} \sigma_{1} \int_{0}^{\bar{x}_{l}\left(\lambda, \sigma_{1}\right)} \mathrm{d} x_{1} k\left(x_{1}\right) \\
& \leq \lambda^{2}\left|q+\left(\frac{1}{2}-\alpha\right) x\right| \int_{0}^{\infty} \mathrm{d} x_{1} k\left(x_{1}\right) \\
& \leq c \lambda^{2}\left(|q|+\left|\frac{1}{2}-\alpha\right| x\right)
\end{aligned}
$$

uniformly on $\sigma$. According to the last line in estimation (39), we study

$$
\begin{aligned}
& \sup _{0 \leq \sigma \leq \bar{\tau}} \int_{0}^{\infty} \mathrm{d} x \mathrm{e}^{-\frac{(x / 2)^{2}}{2 T(\lambda)^{2}}} k(x) \Xi_{\alpha q}(\lambda, \sigma, x) \\
& \leq c^{2} \lambda^{2} q+c\left|\frac{1}{2}-\alpha\right| \int_{0}^{\infty} \mathrm{d} x k(x) \mathrm{e}^{-\frac{(x / 2)^{2}}{2 T(\lambda)^{2}}} \lambda^{2} x
\end{aligned}
$$

The first term goes to zero with velocity $\sim \lambda^{2} q$, whereas the dominated convergence theorem applies to the second term, showing convergence to zero 
uniformly on $q$, with velocity $\sim \lambda^{2-\xi}$ (note that we have supposed $\xi<2$ strictly). This, when put in (39), shows the validity of (38).

A very similar analysis can be done for the remaining sectors $q \lesseqgtr 0$, $|\alpha|<1 / 2$ and $\alpha>1 / 2$. In each case, the net result is that the estimation (41) is always of the form

$$
\sup _{0 \leq \sigma \leq \bar{\tau}} \int_{0}^{\infty} \mathrm{d} x \mathrm{e}^{-\frac{(x / 2)^{2}}{2 T(\lambda)^{2}}} k(x) \Xi_{\alpha q}(\lambda, \sigma, x) \leq C_{1} \lambda^{2}|q|+C_{2} \lambda^{2-\xi}
$$

for suitable real positive constants $C_{1}$ and $C_{2}$. This shows the validity of (38), and thus concludes the proof.

\subsection{Proof of Theorem 3.4}

Let $\mathcal{V}$ be the Banach space of norm continuous $\mathcal{B}_{0}$-valued functions on $[0, \bar{\tau}]$, and let $b \in \mathcal{B}_{0}$. Define the "interaction picture" time rescaled solution of (1)

$$
f_{\lambda}(\tau)=X_{-\lambda^{-2} \tau}^{\lambda} W_{\lambda^{-2} \tau}^{\lambda} b .
$$

Then $f_{\lambda}$ is a solution to the integral equation

$$
f_{\lambda}=b+\mathcal{H}_{\lambda} f_{\lambda},
$$

where the integral operator $\mathcal{H}_{\lambda}$ is defined (recall that $X_{t}^{\lambda}$ is a group of isometries) by

$$
\left(\mathcal{H}_{\lambda} g\right)(\tau)=\lambda^{2} \int_{0}^{\lambda^{-2} \tau} \mathrm{d} s \int_{0}^{s} \mathrm{~d} u X_{s}^{\lambda} A_{01} U_{s-u}^{\lambda} A_{10} X_{u}^{\lambda} g\left(\lambda^{2} u\right) .
$$

In [3], Davies shows that $\mathcal{H}_{\lambda}$ is a Volterra operator. Indeed, by changing coordinates according to (4), (43) can be given an explicit Volterra form, namely as

$$
\left(\mathcal{H}_{\lambda} g\right)(\tau)=\int_{0}^{\tau} \mathrm{d} \sigma X_{-\lambda^{-2} \sigma}^{\lambda} K(\lambda, \tau-\sigma) X_{\lambda^{-2} \sigma}^{\lambda} g(\sigma),
$$

where we defined the "slowly varying" kernel

$$
K(\lambda, \tau)=\int_{0}^{\lambda^{-2} \tau} \mathrm{d} x X_{-x}^{\lambda} A_{01} U_{x}^{\lambda} A_{10}
$$

Because of this reason, and since $K(\lambda, \tau)$ is manifestly bounded by $c$, uniformly on $|\lambda| \leq 1$ thanks to Assumption 3.2, it follows that

$$
\left\|\mathcal{H}_{\lambda}^{n}\right\| \leq c^{n} \bar{\tau}^{n} / n !
$$

and also that the associated von Newmann series expansion

$$
f_{\lambda}=b+\mathcal{H}_{\lambda} b+\mathcal{H}_{\lambda}^{2} b+\cdots
$$

converges. 
We can proceed in similar fashion also for the semigroup (9): iteration gives indeed

$$
\bar{W}_{\lambda^{-2} \tau}^{\lambda}=X_{\lambda^{-2} \tau}^{\lambda}+\int_{0}^{\tau} \mathrm{d} \sigma X_{\lambda^{-2}(\tau-\sigma)}^{\lambda} K_{(\alpha, q, T(\lambda))} W_{\lambda^{-2} \sigma}^{\lambda} .
$$

Accordingly, we define

$$
\bar{f}_{\lambda}(\tau)=X_{-\lambda^{-2} \tau}^{\lambda} \bar{W}_{\lambda^{-2} \tau}^{\lambda} b
$$

so that it follows that $\bar{f}_{\lambda}$ is a solution to the integral equation

$$
\bar{f}_{\lambda}=b+\overline{\mathcal{H}}_{(\lambda \alpha q)} \bar{f}_{\lambda}
$$

where we have defined

$$
\left(\overline{\mathcal{H}}_{(\lambda \alpha q)} g\right)(\tau)=\int_{0}^{\tau} \mathrm{d} \sigma X_{-\lambda^{-2} \sigma}^{\lambda} K_{(\alpha, q, T(\lambda))} X_{\lambda^{-2} \sigma}^{\lambda} g(\sigma) .
$$

Now again, $\overline{\mathcal{H}}_{(\lambda \alpha q)}$ is a Volterra operator, and since $\left\|K_{(\alpha, q, T(\lambda))}\right\| \leq c,(45)$ and (46) follow analogously for $\overline{\mathcal{H}}_{(\lambda \alpha q)}$.

Clearly (see Lemma 8.1), we must show that for any chosen $b \in \mathcal{B}_{0}$,

$$
\sup _{0 \leq t \leq \lambda^{-2} \bar{\tau}}\left\|W_{t}^{\lambda} b-\bar{W}_{t}^{\lambda} b\right\|=\left\|f_{\lambda}-\bar{f}_{\lambda}\right\|_{\infty} \rightarrow 0, \quad \lambda \rightarrow 0 .
$$

We shall do that by defining six suitable Volterra operators, denoted with $\mathcal{H}_{(\lambda \alpha q)}^{(j)}, j=0 \ldots 6$, such that $\mathcal{H}_{(\lambda \alpha q)}^{(0)}=\mathcal{H}_{\lambda}, \mathcal{H}_{(\lambda \alpha q)}^{(N)}=\overline{\mathcal{H}}_{(\lambda \alpha q)}$, for which either we show, according to Lemma 8.1, that

$$
\left\|\mathcal{H}_{(\lambda \alpha q)}^{(j)}-\mathcal{H}_{(\lambda \alpha q)}^{(j-1)}\right\|_{\mathcal{V}} \rightarrow 0, \quad \lambda \rightarrow 0
$$

or more directly that

$$
\left\|f_{(\lambda \alpha q)}^{(j)}-f_{(\lambda \alpha q)}^{(j-1)}\right\|_{\infty} \rightarrow 0, \quad \lambda \rightarrow 0
$$

where

$$
f_{(\lambda \alpha q)}^{(j)}=\sum_{n=0}^{\infty}\left(\mathcal{H}_{(\lambda \alpha q)}^{(j)}\right)^{n} b
$$

is the associated von Neumann series. ${ }^{7}$ Then, our conclusion (see Lemma 8.1) would follow from

$$
\left\|f_{\lambda}-\bar{f}_{\lambda}\right\| \leq \sum_{j=1}^{N}\left\|f_{(\lambda \alpha q)}^{(j)}-f_{(\lambda \alpha q)}^{(j-1)}\right\| \rightarrow 0 \quad \lambda \rightarrow 0 .
$$

To follow our purpose, instead of (4), we perform the coordinate transformations (5) As we noted before, the integration domain $s=0 \ldots \lambda^{-2} \tau$, $u=0 \ldots s$, becomes the domain $\mathcal{D}(\lambda, \tau, \alpha, q)$ in the $(\sigma, x)$-plane defined in (6),

\footnotetext{
7 Note that we have attached the subscript " $(\lambda \alpha q)$ " all throughout: although some Volterra operator may not actually depend on $\alpha$, nor $q$, this unifying notation will become useful in the sequel.
} 
and depicted in Fig. 1. Accordingly, the integral kernel in (43) is now written as

$$
\begin{aligned}
\left(\mathcal{H}_{\lambda} g\right)(\tau)= & \iint_{\mathcal{D}(\lambda, \tau, \alpha, q)} \mathrm{d} \sigma \mathrm{d} x X_{-\lambda^{-2} \sigma+q-}^{\lambda}\left(\alpha+\frac{1}{2}\right) x \\
& \times g\left(\sigma-\lambda_{01} U_{x}^{\lambda} A_{10} X_{\lambda^{-2} \sigma-q-}^{\lambda}(q+(1 / 2-\alpha) x\right. \\
& \times g)) .
\end{aligned}
$$

We shall work throughout with the choice $\alpha<-1 / 2$ and $q>0$, without loss of generality (the structure of the proof is basically the same for all the remaining sectors, and actually the case $|\alpha|<1 / 2$ presents less difficulties).

The first thing we shall be concerned with, is to find a way to substitute the free "polarization evolution" $P_{1} U_{x}$ in place of the interacting $P_{1} U_{x}^{\lambda}$ in the middle of the kernel in (49). This step is accomplished by defining a related integral operator $\mathcal{H}_{\lambda}^{(1)}$ by

$$
\begin{aligned}
\left(\mathcal{H}_{(\lambda \alpha q)}^{(1)} g\right)(\tau)= & \iint_{\mathcal{D}_{(1)}(\lambda, \tau, \alpha, q)} \mathrm{d} \sigma \mathrm{d} x X_{-\lambda^{-2} \sigma+q-\left(\alpha+\frac{1}{2}\right) x}^{\lambda} A_{01} U_{x} A_{10} X_{\lambda^{-2} \sigma-q+\left(\alpha-\frac{1}{2}\right) x}^{\lambda} \\
& \times g\left(\sigma-\lambda^{2}(q+(1 / 2-\alpha) x)\right) .
\end{aligned}
$$

Here, we have denoted $\mathcal{D}_{(1)}=\mathcal{D}$ for sake of notation. Note that $\mathcal{H}_{(\lambda \alpha q)}^{(1)}$ does not depend on $\alpha$, nor on $q$, although both the latter parameters appear in its definition. To compare the two integral operators, take a bounded $g \in \mathcal{V}$ and estimate

$$
\begin{aligned}
\left\|\left(\mathcal{H}_{\lambda}-\mathcal{H}_{(\lambda \alpha q)}^{(1)}\right) g\right\| & \leq \underset{\mathcal{D}(\lambda, \tau, \alpha, q)}{\iint_{0} \mathrm{~d} \sigma \mathrm{d} x\left\|A_{01}\left(U_{x}^{\lambda}-U_{x}\right) A_{10}\right\|\|g\|_{\infty}} \\
& \leq \max \{1,|1 / 2-\alpha|\} \bar{\tau}\|g\|_{\infty} \int_{0}^{\lambda^{-2} \bar{\tau}} \mathrm{d} x\left\|A_{01}\left(U_{x}^{\lambda}-U_{x}\right) A_{10}\right\|
\end{aligned}
$$

(note for later purposes that the estimation does not depend on $q$ ). Our convergence hypothesis 3.3 on $U_{x}^{\lambda}$ allows then to conclude that this goes to zero when $\lambda \rightarrow 0$ uniformly on every $\|g\|=1$, so that we obtain

$$
\lim _{\lambda \rightarrow 0}\left\|\mathcal{H}_{\lambda}-\mathcal{H}_{(\lambda \alpha q)}^{(1)}\right\|=0
$$

We proceed along similar lines to smooth the kernel with $T(\lambda)$ : define

$$
\begin{aligned}
\left(\mathcal{H}_{(\lambda \alpha q)}^{(2)} g\right)(\tau) & =\iint_{\mathcal{D}_{(2)}(\lambda, \tau, \alpha, q)} \mathrm{d} \sigma \mathrm{d} x \mathrm{e}^{-\frac{(x / 2)^{2}}{2 T(\lambda)^{2}}} X_{-\lambda{ }^{-2} \sigma+q-\left(\alpha+\frac{1}{2}\right) x}^{\lambda} A_{01} U_{x} A_{10} X_{\lambda^{-2} \sigma-q+\left(\alpha-\frac{1}{2}\right) x}^{\lambda} \\
& \times g\left(\sigma-\lambda^{2}(q+(1 / 2-\alpha) x)\right) .
\end{aligned}
$$


Here we have denoted $\mathcal{D}_{(2)}=\mathcal{D}_{(1)}$. To compare with $\mathcal{H}_{(\lambda \alpha q)}^{(1)}$, take a bounded $g \in \mathcal{V}$ and estimate

$$
\begin{aligned}
& \left\|\left(\mathcal{H}_{(\lambda \alpha q)}^{(1)}-\mathcal{H}_{(\lambda \alpha q)}^{(2)}\right) g\right\| \leq \iint_{\mathcal{D}(\lambda, \tau, \alpha, q)} \mathrm{d} \sigma \mathrm{d} x\left|1-\mathrm{e}^{-\frac{(x / 2)^{2}}{2 T(\lambda)^{2}}}\right|\left\|A_{01} U_{x} A_{10}\right\|\|g\|_{\infty} \\
& \quad \leq \max \left\{1,\left|\frac{1}{2}-\alpha\right|\right\} \bar{\tau}\|g\|_{\infty} \int_{0}^{\infty} \mathrm{d} x\left|1-\mathrm{e}^{-\frac{(x / 2)^{2}}{2 T(\lambda)^{2}}}\right|\left\|A_{01} U_{x} A_{10}\right\|
\end{aligned}
$$

Hypothesis 3.2 furnishes an integrable upper bound to the last integrand, and so the integral goes to zero in the limit $\lambda \rightarrow 0$ because of the dominated convergence theorem: in fact, one has pointwise convergence

$$
\left|1-\mathrm{e}^{-\frac{(x / 2)^{2}}{2 T(\lambda)^{2}}}\right| \rightarrow 0, \quad \lambda \rightarrow 0
$$

due to our hypothesis $0<\xi$. Uniform convergence on all $\|g\|=1$ in the last line of our estimation then shows that

$$
\lim _{\lambda \rightarrow 0}\left\|\mathcal{H}_{(\lambda \alpha q)}^{(1)}-\mathcal{H}_{(\lambda \alpha q)}^{(2)}\right\|=0 .
$$

We note that $\mathcal{H}_{(\lambda \alpha q)}^{(2)}$, as $\mathcal{H}_{(\lambda \alpha q)}^{(1)}$ and $\mathcal{H}_{\lambda}$, is also a Volterra operator, as is easy to verify: it would suffice to apply the inverse transform (5) for the specific choice of $\alpha$ and $q$, and subsequently apply the transform (5) for $q=0$ and $\alpha=1 / 2$, to find $\mathcal{H}_{(\lambda \alpha q)}^{(2)}$ into its explicit Volterra form (and also find that it does not depend on $(\alpha, q))$.

Now define the restricted domain (see Fig. 1)

$$
\mathcal{D}_{(3)}(\lambda, \tau, \alpha, q)=\mathcal{D}_{(2)}(\lambda, \tau, \alpha, q) \cap[0, \tau] \times[0, \infty)
$$

and define $\mathcal{H}_{(\lambda \alpha q)}^{(3)}$ accordingly, as the restriction of $\mathcal{H}_{(\lambda \alpha q)}^{(2)}$ to $\mathcal{D}_{(3)}(\lambda, \tau, \alpha, q)$, that is,

$$
\begin{aligned}
\left(\mathcal{H}_{(\lambda \alpha q)}^{(3)} g\right)(\tau)= & \iint_{\mathcal{D}_{(3)}(\lambda, \tau, \alpha, q)} \mathrm{d} \sigma \mathrm{d} x \mathrm{e}^{-\frac{(x / 2)^{2}}{2 T(\lambda)^{2}} X_{-\lambda}^{\lambda}-2 \sigma+q-\left(\alpha+\frac{1}{2}\right) x} A_{01} U_{x} A_{10} X_{\lambda^{-2} \sigma-q+\left(\alpha-\frac{1}{2}\right) x}^{\lambda} \\
& \times g\left(\sigma-\lambda^{2}(q+(1 / 2-\alpha) x)\right) .
\end{aligned}
$$

This is again a Volterra operator, as the image of $\mathcal{D}_{(3)}(\lambda, \tau, \alpha, q)$ under the composition of (5) and its inverse for $q=0$ and $\alpha=1 / 2$, is inside the rectangular triangle, image of $\mathcal{D}_{(2)}(\lambda, \tau, \alpha, q)$ through the same transformations. So the composition of (5) its inverse for $q=0$ and $\alpha=1 / 2$, will put both $\mathcal{H}_{(\lambda \alpha q)}^{(2)}$ and $\mathcal{H}_{(\lambda \alpha q)}^{(3)}$ in their explicit Volterra form.

We must prove that

$$
\lim _{\lambda \rightarrow 0}\left\|\mathcal{H}_{(\lambda \alpha q)}^{(2)}-\mathcal{H}_{(\lambda \alpha q)}^{(3)}\right\| \rightarrow 0
$$

(note that for $|\alpha|<1 / 2$ this is trivial, as one has $\mathcal{H}_{(\lambda \alpha q)}^{(2)}=\mathcal{H}_{(\lambda \alpha q)}^{(3)}$, for the upper vertex of the triangular domain projects on the horizontal edge of the 
latter (the one lying on the $\sigma$-axis), and thus $\mathcal{D}_{(2)}(\lambda, \tau, \alpha, q)=\mathcal{D}_{(3)}(\lambda, \tau, \alpha, q)$ ). To this end, we take as usual any $g \in \mathcal{V}$ with $\|g\|=1$ and estimate

$$
\begin{aligned}
& \left\|\left(\left(\mathcal{H}_{\lambda}^{(2)}-\mathcal{H}_{(\lambda \alpha q)}^{(3)}\right) g\right)(\tau)\right\| \leq \int_{\mathcal{D}(\lambda, \tau, \alpha, q) \cap\left\{\tau \leq \sigma \leq|1 / 2-\alpha| \tau+\lambda^{2} q\right\}} \mathrm{d} \sigma \mathrm{d} x\left\|A_{01} U_{x} A_{10}\right\|\|g\|_{\infty} \\
& \quad \leq\|g\|_{\infty}\left\{\lambda^{2} q \int_{0}^{\infty} \mathrm{d} x\left\|A_{01} U_{x} A_{10}\right\|+\int_{\tau+\lambda^{2} q}^{\left(\frac{1}{2}-\alpha\right) \tau+\lambda^{2} q} \mathrm{~d} \sigma \int_{\bar{x}_{r}(\lambda, q, \sigma)}^{\infty} \mathrm{d} x\left\|A_{01} U_{x} A_{10}\right\|\right\}
\end{aligned}
$$

Here

$$
\bar{x}_{r}(\lambda, q, \sigma)=\frac{q+\lambda^{-2}(\tau-\sigma)}{\alpha+\frac{1}{2}}
$$

is the $x$-coordinate of the right edge of the triangle $\mathcal{D}(\lambda, \tau, \alpha, q)$ (see Fig. 1 ). The first term in the curly brackets clearly goes to zero uniformly on $\tau$ (with speed $\sim \lambda^{-2} q$ ), because of our boundedness hypothesis 3.2. In the second term in the curly brackets, we change coordinates according to $\sigma \rightarrow \sigma-\lambda^{2} q$, so that it becomes equal to

$$
\int_{\tau}^{\left(\frac{1}{2}-\alpha\right) \tau} \mathrm{d} \sigma \int_{\bar{x}_{r}(\lambda, 0, \sigma)}^{+\infty} \mathrm{d} x\left\|A_{01} U_{x} A_{10}\right\| .
$$

But this converges to zero as $\lambda \rightarrow 0$, as a consequence of $\bar{x}_{r}(\lambda, 0, \sigma) \rightarrow$ $\infty, \quad \lambda \rightarrow 0$, of Assumption 3.2, and of the boundedness of the $\sigma$-integration domain. Uniform convergence on all $0 \leq \tau \leq \bar{\tau}$ (and real $q$ ) follows from the fact that the $\sigma$-integration domain is compact. This shows the validity of (53). We now define the following "time localized" Volterra operator:

$$
\begin{aligned}
\left(\mathcal{H}_{(\lambda \alpha q)}^{(4)} g\right)(\tau) & =\iint_{\mathcal{D}_{(4)}(\lambda, \tau, \alpha, q)} \mathrm{d} \sigma \mathrm{d} x \mathrm{e}^{-\frac{(x / 2)^{2}}{2 T(\lambda)^{2}} X_{-\lambda}^{\lambda}-2 \sigma+q-\left(\alpha+\frac{1}{2}\right) x} A_{01} U_{x} A_{10} X_{\lambda-2}^{\lambda} \sigma-q+\left(\alpha-\frac{1}{2}\right) x \\
& \times g(\sigma)
\end{aligned}
$$

where we have put $\left.\mathcal{D}_{(4)}(\lambda, \tau, \alpha, q)\right)=\mathcal{D}_{(3)}(\lambda, \tau, \alpha, q)$. It turns out that proving an operator convergence to $\mathcal{H}_{(\lambda \alpha q)}^{(3)}$ is impossible, due to the strong requirement of uniform convergence with respect to any normalized $g \in \mathcal{V}$. However, the Volterra operators $\mathcal{H}_{(\lambda \alpha q)}^{(3)}$ and $\mathcal{H}_{(\lambda \alpha q)}^{(4)}$ do fulfill the hypotheses of Lemma 8.2, and so we conclude that

$$
\lim _{\lambda \rightarrow 0}\left\|f_{(\lambda \alpha q)}^{(3)}-f_{(\lambda \alpha q)}^{(4)}\right\|=0
$$

We now go back to consider the domain $\mathcal{D}_{(4)}(\lambda, \tau, \alpha, q)$ as a function of $\lambda$, and note that it tends to fill the strip

$$
\mathcal{D}_{(5)}(\tau)=[0, \tau] \times[0, \infty]
$$


Accordingly, we define the following Volterra integral operator on this strip: $\mathcal{H}_{(\lambda \alpha q)}^{(5)} g=\int_{0}^{\tau} \mathrm{d} \sigma \int_{0}^{\infty} \mathrm{d} x \mathrm{e}^{-\frac{(x / 2)^{2}}{2 T(\lambda)^{2}}} X_{-\lambda^{-2} \sigma+q-\left(\alpha+\frac{1}{2}\right) x}^{\lambda} A_{01} U_{x} A_{10} X_{\lambda^{-2} \sigma-q+\left(\alpha-\frac{1}{2}\right) x}^{\lambda} g(\sigma)$. and note that

$$
\begin{aligned}
& \left\|\left(\left(\mathcal{H}_{(\lambda \alpha q)}^{(5)}-\mathcal{H}_{(\lambda \alpha q)}^{(4)}\right) g\right)(\tau)\right\| \leq \iint_{\mathcal{D}^{(5)}(\tau) \backslash \mathcal{D}^{(4)}(\lambda, \tau, \alpha, q)} \mathrm{d} \sigma \mathrm{d} x\left\|A_{01} U_{x} A_{10}\right\|\|g\|_{\infty} \\
& \quad \leq\|g\|_{\infty}\left\{\lambda^{2} q \int_{0}^{\infty} \mathrm{d} x\left\|A_{01} U_{x} A_{10}\right\|+\int_{\lambda^{2} q}^{\tau} \mathrm{d} \sigma \int_{\bar{x}_{l}(\lambda, q, \sigma)}^{\infty} \mathrm{d} x\left\|A_{01} U_{x} A_{10}\right\|\right\}
\end{aligned}
$$

where

$$
\bar{x}_{l}(\lambda, q, \sigma)=\frac{q-\lambda^{-2} \sigma}{\alpha-\frac{1}{2}}
$$

is the $x$-coordinate of the left edge of the triangular domain $\mathcal{D}(\lambda, \tau, \alpha, q)$ (see Fig. 1). Now the first term in the curly brackets clearly goes to zero as $\lambda \rightarrow 0$ (with velocity $\sim \lambda^{2} q$ ), due to hypothesis 3.2 for $\lambda=0$. In the second one, as before, we change coordinate according to $\sigma \rightarrow \sigma-\lambda^{2} q$, obtaining

$$
\int_{0}^{\tau-\lambda^{2} q} \mathrm{~d} \sigma \int_{\bar{x}_{l}(\lambda, 0, \sigma)}^{\infty} \mathrm{d} x\left\|A_{01} U_{x} A_{10}\right\| \leq \int_{0}^{\tau} \mathrm{d} \sigma \int_{\bar{x}_{l}(\lambda, 0, \sigma)}^{\infty} \mathrm{d} x\left\|A_{01} U_{x} A_{10}\right\| .
$$

This last term can be seen to go to zero uniformly on $0 \leq \tau \leq \bar{\tau}$ (and real $q$ ) by arguing, as before, that $\bar{x}_{l}(\lambda, 0, \sigma) \rightarrow \infty, \lambda \rightarrow 0$ for a.e. $\sigma \in[0, \tau]$, and using hypothesis 3.2 . So it follows that

$$
\left\|\mathcal{H}_{(\lambda \alpha q)}^{(4)}-\mathcal{H}_{(\lambda \alpha q)}^{(5)}\right\| \rightarrow 0, \quad \lambda \rightarrow 0 .
$$

Now we can finally compare with $\mathcal{H}_{(\lambda \alpha q)}^{(6)}:=\overline{\mathcal{H}}_{(\lambda \alpha q)}$. By adding and subtracting obvious terms, we estimate

$$
\begin{aligned}
\left\|\left(\mathcal{H}_{(\lambda \alpha q)}^{(5)}-\overline{\mathcal{H}}_{(\lambda \alpha q)}\right) g\right\| \leq & \bar{\tau} \int_{0}^{\infty} \mathrm{d} x\left\|X_{q-\left(\alpha+\frac{1}{2}\right) x}^{\lambda}-P_{0} U_{q-\left(\alpha+\frac{1}{2}\right) x}\right\|\left\|A_{01} U_{x} A_{10}\right\|\|g\|_{\infty} \\
& +\bar{\tau} \int_{0}^{\infty} \mathrm{d} x\left\|A_{01} U_{x} A_{10}\right\| \| X_{\left(\alpha-\frac{1}{2}\right) x-q}^{\lambda} \\
& -P_{0} U_{\left(\alpha-\frac{1}{2}\right) x-q}\|\| g \|_{\infty} .
\end{aligned}
$$

Uniform convergence to zero follows by hypothesis 3.2 together with the dominated convergence theorem, using the fact that for every $x \in \mathbb{R}$

$$
\lim _{\lambda \rightarrow 0}\left\|X_{x}^{\lambda}-P_{0} U_{x}\right\|=0 .
$$

This proves the estimation in (48) and thus finishes the proof. 


\subsection{Proof of Theorem 4.2}

We shall borrow most part of the proof of Theorem 3.4. Accordingly, we should denote for example with $\mathcal{H}_{(\lambda q)}^{(j)}$ the operator defined in Theorem 3.4 as $\mathcal{H}_{(\lambda 0 q)}^{(j)}$, $\left.\mathcal{D}_{(} \lambda, \tau, q\right) \equiv \mathcal{D}(\lambda, \tau, \alpha, q)$ and so on. Define

$$
\mathcal{H}_{\lambda}^{(j)}=\frac{1}{\sqrt{2 \pi} T(\lambda)} \int_{-\infty}^{\infty} \mathrm{d} q \mathrm{e}^{-\frac{q^{2}}{2 T(\lambda)^{2}}} \mathcal{H}_{(\lambda q)}^{(j)} .
$$

This is obviously Volterra, being an integral of Volterra operators. A closer inspection soon reveals that also

$$
\left\|\mathcal{H}_{\lambda}^{(j)}\right\| \leq \frac{1}{\sqrt{2 \pi} T(\lambda)} \int_{-\infty}^{\infty} \mathrm{d} q \mathrm{e}^{-\frac{q^{2}}{2 T(\lambda)^{2}}}\left\|\mathcal{H}_{(\lambda q)}^{(j)}\right\| \leq c \bar{\tau}
$$

as for each $j, \mathcal{H}_{(\lambda q)}^{(j)}$ is bounded by $c$ uniformly on $q$ and

$$
\frac{1}{\sqrt{2 \pi} T(\lambda)} \int_{-\infty}^{\infty} \mathrm{d} q \mathrm{e}^{-\frac{q^{2}}{2 T(\lambda)^{2}}}=1
$$

We proceed on the very same lines of Theorem 3.4: the proofs that

$$
\lim _{\lambda \rightarrow 0}\left\|\mathcal{H}_{\lambda}^{(j)}-\mathcal{H}_{\lambda}^{(j-1)}\right\|=0
$$

for $j=1,2$, and $j=6$ are in fact identical to that of the foretold Theorem, as, for those values for $j$, one has

$$
\lim _{\lambda \rightarrow 0}\left\|\mathcal{H}_{(\lambda, q)}^{(j)}-\mathcal{H}_{(\lambda, q)}^{(j-1)}\right\|=0
$$

uniformly on $q$. Normalization (58) can be exploited to state the validity of the above relation for $j=3$ and $j=5$. In fact, for these values of $j$, we can estimate

$$
\left\|\mathcal{H}_{\lambda}^{(j)}-\mathcal{H}_{\lambda}^{(j-1)}\right\| \leq \frac{1}{\sqrt{2 \pi} T(\lambda)} \int_{-\infty}^{\infty} \mathrm{d} q \mathrm{e}^{-\frac{q^{2}}{2 T(\lambda)^{2}}}\left\|\mathcal{H}_{(\lambda, q)}^{(j)}-\mathcal{H}_{(\lambda, q)}^{(j-1)}\right\| .
$$

Now, the norm in the integrand goes to zero as $\sim c_{1}^{(j)}(\lambda)+c_{2}^{(j)} \lambda^{2} q$, with $c_{1}^{(j)}(\lambda) \rightarrow 0$ uniformly on $q$ as $\lambda \rightarrow 0$, as already noted in the proof of Theorem 3.4 , so the whole integral goes to zero as $\lambda \rightarrow 0$ precisely because $T(\lambda)$ scales with $|\lambda|^{-\xi}$ and $\xi<2$ by hypothesis.

It remains to show that if $f_{\lambda}^{(j)}=\sum_{n}\left(\mathcal{H}_{\lambda}^{(j)}\right)^{n} b$, for some initial condition $b \in \mathcal{B}_{0}$, then

$$
\lim _{\lambda \rightarrow 0}\left\|f_{\lambda}^{(4)}-f_{\lambda}^{(3)}\right\|=0
$$


Proceeding according to Lemma 8.2, we perform a telescopic expansion and estimate

$$
\begin{aligned}
\left\|f_{\lambda}^{(3)}-f_{\lambda}^{(4)}\right\|_{\infty} \leq & \sum_{n=1}^{\infty} \sum_{l=1}^{n-1} \frac{(\bar{\tau} c)^{n-l-1}}{(n-l) !} \frac{1}{\sqrt{2 \pi} T(\lambda)} \int_{-\infty}^{\infty} \mathrm{d} q \mathrm{e}^{-\frac{q^{2}}{2 T(\lambda)^{2}}} \\
& \times\left\|\left(\mathcal{H}_{(\lambda q)}^{(3)}-\mathcal{H}_{(\lambda q)}^{(4)}\right)\left(\mathcal{H}_{\lambda}^{(4)}\right)^{l} b\right\|_{\infty} .
\end{aligned}
$$

Now we compute

$$
\begin{aligned}
& {\left[\left(\mathcal{H}_{(\lambda q)}^{(3)}-\mathcal{H}_{(\lambda q)}^{(4)}\right)\left(\mathcal{H}_{\lambda}^{(4)}\right)^{l} b\right](\tau)} \\
& =\iint_{\overline{\mathcal{D}}(\lambda, \tau, q)} \mathrm{d} \sigma \mathrm{d} x \mathrm{e}^{-\frac{(x / 2)^{2}}{2 T(\lambda)^{2}}} K_{q}(\lambda, \sigma, x)\left\{\left[\left(\mathcal{H}_{\lambda}^{(4)}\right)^{l} b\right]\left(\sigma-\lambda^{2}(q+x / 2)\right)-\left[\left(\mathcal{H}_{\lambda}^{(4)}\right)^{l} b\right](\sigma)\right\} \\
& =\frac{1}{\sqrt{2 \pi} T(\lambda)} \int_{-\infty}^{\infty} \mathrm{d} q^{\prime} \mathrm{e}^{-\frac{q^{2}}{2 T(\lambda)^{2}}} \iint_{\overline{\mathcal{D}}(\lambda, \tau, q)} \mathrm{d} \sigma \mathrm{d} x \mathrm{e}^{-\frac{(x / 2)^{2}}{2 T(\lambda)^{2}}} K_{q}(\lambda, \sigma, x) \\
& \quad \times\left\{\left[\mathcal{H}_{\lambda q^{\prime}}^{(4)}\left(\left(\mathcal{H}_{\lambda}^{(4)}\right)^{l-1} b\right)\right]\left(\sigma-\lambda^{2}(q+x / 2)\right)-\left[\mathcal{H}_{\lambda q^{\prime}}^{(4)}\left(\left(\mathcal{H}_{\lambda}^{(4)}\right)^{l-1} b\right)\right](\sigma)\right\} \\
& =\frac{1}{\sqrt{2 \pi} T(\lambda)} \int_{-\infty}^{\infty} \mathrm{d} q^{\prime} \mathrm{e}^{-\frac{q^{2}}{2 T(\lambda)^{2}}} \iint_{\overline{\mathcal{D}}(\lambda, \tau, q)} \mathrm{d} \sigma \mathrm{d} x \mathrm{e}^{-\frac{(x / 2)^{2}}{2 T(\lambda)^{2}}} K_{q}(\lambda, \sigma, x) \\
& \quad \times \quad \iint_{\left(\sigma_{1}, x_{1}\right) \mathrm{e}^{-\frac{(x / 2)^{2}}{2 T(\lambda)^{2}}} K_{q^{\prime}}\left(\lambda, \sigma_{1}, x_{1}\right)\left[\left(\mathcal{H}_{\lambda}^{(4)}\right)^{l-1} b\right]\left(\sigma_{1}\right)}^{\mathrm{d} \sigma_{1} \mathrm{~d} x_{1} \Delta^{\lambda}\left(\sigma^{\prime}\right)} \\
& \quad \tilde{S}\left(\lambda, q, q^{\prime}, \sigma, x\right)
\end{aligned}
$$

where we have put $\Delta^{\lambda}=\chi_{1}^{\lambda}-\chi_{2}^{\lambda}, \chi_{1}^{\lambda}$ being the characteristic function of $\overline{\mathcal{D}}\left(\lambda, \sigma-\lambda^{2}(q+x / 2), q^{\prime}\right), \chi_{2}^{\lambda}$ the characteristic function of $\overline{\mathcal{D}}\left(\lambda, \sigma, q^{\prime}\right)$, and we have defined

$$
\widetilde{S}\left(\lambda, q, q^{\prime}, \sigma, x\right)=\overline{\mathcal{D}}\left(\lambda, \sigma-\lambda^{2}(q+x / 2), q^{\prime}\right) \cup \overline{\mathcal{D}}\left(\lambda, \sigma, q^{\prime}\right) .
$$

Passing to the norms we obtain

$$
\begin{aligned}
& \left\|\left(\mathcal{H}_{(\lambda q)}^{(3)}-\mathcal{H}_{(\lambda q)}^{(4)}\right) \mathcal{H}_{\lambda}^{(4)^{l}} b\right\|_{\infty} \leq \frac{(\bar{\tau} c)^{l-1}}{(l-1) !}\|b\|_{\mathcal{B}_{0}} \frac{1}{\sqrt{2 \pi} T(\lambda)} \int_{-\infty}^{\infty} \mathrm{d} q^{\prime} \mathrm{e}^{-\frac{q^{2}}{2 T(\lambda)^{2}}} \\
& \quad \times \sup _{0<\tau<\bar{\tau}} \iint \mathrm{d} \sigma \mathrm{d} x \mathrm{e}^{-\frac{(x / 2)^{2}}{2 T(\lambda)^{2}}} k(x) \Xi_{q q^{\prime}}(\lambda, \sigma, x) \\
& \mathcal{D}_{(\lambda, \tau, q)}
\end{aligned}
$$

where the slight modification of the related definition of $\Xi_{q}(\lambda, \sigma, x)$ in (40) is given by

$$
\Xi_{q q^{\prime}}(\lambda, \sigma, x)=\iint_{\widetilde{S}\left(\lambda, q, q^{\prime}, \sigma, x\right)} \mathrm{d} \sigma_{1} \mathrm{~d} x_{1}\left|\Delta^{\lambda}\left(\sigma_{1}, x_{1}\right)\right| k\left(x_{1}\right) .
$$


Proceeding on the same lines as in Lemma 8.2 we find the asymptotic behavior

$$
\begin{aligned}
& \sup _{0<\tau<\bar{\tau}} \iint_{\mathcal{D}(\lambda, \tau, q)} \mathrm{d} \sigma \mathrm{d} x \mathrm{e}^{-\frac{(x / 2)^{2}}{2 T(\lambda)^{2}}} k(x) \Xi_{q q^{\prime}}(\lambda, \sigma, x) \\
& \leq \bar{\tau} \sup _{0 \leq \sigma \leq \bar{\tau}} \int_{0}^{\infty} \mathrm{d} x \mathrm{e}^{-\frac{(x / 2)^{2}}{2 T(\lambda)^{2}}} k(x) \Xi_{q q^{\prime}}(\lambda, \sigma, x) \\
& \leq \bar{\tau}\left\{c_{1}(\lambda)+c_{2} \lambda^{2}|q|+c_{3} \lambda^{2}\left|q^{\prime}\right|\right\}
\end{aligned}
$$

where $c_{1}(\lambda) \rightarrow 0$ uniformly on $q$ and $q^{\prime}$. This, plus the fact that $\xi<2$ by our hypothesis, allows us conclude that (60) holds, as can be seen by putting result (64) into (62), and then back into (61), and by using the dominated convergence theorem. Collecting the results as in Theorem 3.4 concludes the proof.

\section{Acknowledgements}

We wish to thank Prof. Fausto Rossi (Phys. Dept., Politecnic of Turin), Prof. Hisao Fujita Yashima and Prof Enrico Priola (Math. Dept., University of Turin), Prof. Claude-Alain Pillet (Centre de Physique Théorique, Marseille), Prof. Vladimir Gritsev (Phys. Dept., University of Fribourg) and finally Dr. Taj Mohammad (Phys. Dept., University of Turin) for precious help and stimulating discussions.

\section{References}

[1] Van Hove, L.: Energy corrections and persistent perturbation effects in continuous spectra. Physica 21(6-10), 901-923 (1955)

[2] Davies, E.B.: Markovian master equations. Commun. Math. Phys. 39, 91-110 (1974)

[3] Davies, E.B.: Markovian master equations. II. Math. Ann. 219, 147-158 (1976)

[4] Alicki, R.: The Markov master equations and the Fermi golden rule. Int. J. Theor. Phys. 16(5), 351-355 (1977)

[5] Fermi, E.: Nuclear Physics, chap. 2-3, Revised edn., University of Chicago Press, Chicago (1950)

[6] Taj, D., Genovese, L., Rossi, F.: Quantum-transport simulations with the Wigner-function formalism: Failure of conventional boundary-condition schemes. Europhys. Lett. 74(6), 1060-1066 (2006)

[7] Attal, S., Joye, A., Pillet, C.-A.: Open Quantum Systems I, II and III, vol. 1880-1882. LNM (2006)

[8] Davies, E.B.: Quantum Theory of Open Systems. Academic Press, London (1976)

[9] Umanità, V.: Classification and decomposition of Quantum Markov Semigroups. Probab. Theory Relat. Fields 134(4), 603-623 (2006) 
[10] Fagnola, F.: Quantum Markov Semigroups and Quantum Flows. Proyecciones 18(3), 1-144 (1999)

[11] Dümcke, R., Spohn, H.: The Proper Form of the Generator in the Weak Coupling Limit. Z. Physik B 34, 419-422 (1979)

[12] Taj, D., Lotti, R.C., Rossi, F.: Microscopic modeling of energy relaxation and decoherence in quantum optoelectronic devices at the nanoscale. Eur. Phys. J. B 72, 305322 (2009) (Colloquium)

[13] Taj, D., Rossi, F.: Completely positive Markovian quantum dynamics in the weak-coupling limit. Phys. Rev. A 78, 052113 (2008)

[14] Nakajima, S.: On Quantum Theory of Transport Phenomena. Prog. Theor. Phys. 20(6), 948-959 (1958)

[15] Zwanzig, R.: Ensemble Method in the Theory of Irreversibility. J. Chem. Phys. 33, 1338 (1960)

[16] Yosida, K.: Functional Analysis. Springer, Berlin (1968)

[17] Goldstein, J.: A Lie product formula for one parameter groups of isometries on Banach spaces. Math. Ann. 186, 299-306 (1970)

[18] Bratteli, O., Robinson, D.W.: Operator Algebras and Quantum Statistical Mechanics I, 2nd edn. Springer, Berlin (2002)

[19] Chernoff, P.R.: Note on product formulae for operator semigroups. J. Funct. Anal. 2, 238-242 (1968)

[20] Sakai, S.: $C^{*}$-algebras and $W^{*}$-algebras. Springer, Berlin (1998)

[21] Lindblad, G.: On the Generators of Quantum Dynamical Semigroups. Commun. Math. Phys. 48, 119-130 (1976)

[22] Kraus, K.: General state changes in quantum theory. Ann. Phys. 64, 311-335 (1970)

David Taj

Physics Department

University of Fribourg

Ch. du Musée 3

1700 Fribourg

Switzerland

e-mail: david.taj@unifr.ch; david.taj@gmail.com

Communicated by Jens Marklof.

Received: April 26, 2010.

Accepted: September 13, 2010. 